\title{
Fischer Indole Reaction in Batch and Flow Employing a Sulfonic Acid Resin: Synthesis of Pyrido[2,3-a]carbazoles
}

Caroline Bosch, ${ }^{\dagger}$ Pablo López-Lledó, ${ }^{\dagger}$ Josep Bonjoch ${ }^{\star,},{ }^{\dagger}$ Ben Bradshaw, ${ }^{\dagger}$ Pieter J. Nieuwland ${ }^{\ddagger}$, Daniel Blanco-Ania ${ }^{\S}$, and Floris P. J. T. Rutjes ${ }^{\star, \S}$

\footnotetext{
+ Laboratori de Química Orgànica, Facultat de Farmàcia, IBUB, Universitat de Barcelona, Av. Joan XXIII s/n, 08028-Barcelona, Spain

${ }^{\ddagger}$ FutureChemistry Holding BV, Toernooiveld 100, 6525 EC Nijmegen, The Netherlands

$\S$ Institute for Molecules and Materials, Radboud University, Heyendaalseweg 135, 6525 AJ Nijmegen, The Netherlands

e-mail address: josep.bonjoch@ub.edu; F.Rutjes@science.ru.nl
}

\section{Contents}

$\begin{array}{ll}\text { General information } & \text { S2 }\end{array}$

$\begin{array}{ll}\text { Experimental procedures } & \text { S3 }\end{array}$

$\begin{array}{ll}\text { NMR tables of all compounds } & \text { S14 }\end{array}$

${ }^{1} \mathrm{H},{ }^{13} \mathrm{C}-\mathrm{NMR}$ spectra $\quad \mathrm{S} 18$

$\begin{array}{ll}\text { X-ray data for (4a) } & \text { S38 }\end{array}$ 


\section{GENERAL INFORMATION}

All reactions were carried out under an argon atmosphere in dry solvents under anhydrous conditions. Drying of organic extracts during workup of reactions was performed over anhydrous $\mathrm{Na}_{2} \mathrm{SO}_{4}$ except where otherwise stated. Evaporation of solvents was accomplished with a rotatory evaporator. Analytical thin layer chromatography was performed on $\mathrm{SiO}_{2}$ (Merck silica gel $60 \mathrm{~F}_{254}$ ) or on glass-backed plates pre-coated with silica and the spots were located with aqueous $\mathrm{KMnO}_{4}$ or $p$-anisaldehyde. Chromatography refers to flash chromatography and was carried out on $\mathrm{SiO}_{2}$ (silica gel $60 \mathrm{ACC}, 35-75 \mu \mathrm{m}, 230-240$ mesh ASTM). NMR spectra were recorded in $\mathrm{CDCl}_{3}$ on a Varian Mercury $400 \mathrm{MHz}$ or Varian VNMRS $400 \mathrm{MHz}$. Chemical shifts of ${ }^{1} \mathrm{H}$ and ${ }^{13} \mathrm{C}$ NMR spectra are reported in ppm downfield $(\delta)$ from $\mathrm{Me}_{4} \mathrm{Si}$. All NMR data assignments are supported by COSY and HSQC experiments.

Melting points were performed on recrystallized solids and are uncorrected. 


\section{EXPERIMENTAL PROCEDURES}

\section{Batch Reactions}

\section{General procedure A:}<smiles>[R]c1ccc(NN)cc1</smiles><smiles>O=C1CCCCC1</smiles>

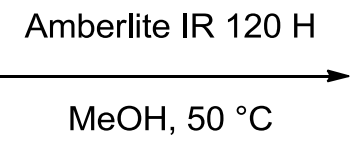<smiles>[R]c1ccc2[nH]c3c(c2c1)CCCC3</smiles>

To a stirring solution of cyclohexanone (1 equiv) and solid acid catalyst Amberlite IR $120 \mathrm{H}^{\circledR}(5$ equiv w/w) in $\mathrm{MeOH}(0.1-0.5 \mathrm{M})$ at $50{ }^{\circ} \mathrm{C}$, was added phenylhydrazine 1 or phenylhydrazine hydrochloric salt $1 \cdot \mathrm{HCl}$ (1.1 equiv). The mixture was left stirring at $50{ }^{\circ} \mathrm{C}$ for the indicated time. After cooling, the reaction mixture was filtered and the resin was washed with $\mathrm{CH}_{2} \mathrm{Cl}_{2}$ and $\mathrm{MeOH}$ and the crude product was purified by crystallization from $\mathrm{MeOH} / \mathrm{H}_{2} \mathrm{O}$ to afford the pure carbazole.

\section{2,3,4,9-Tetrahydro-1H-carbazole (2a)}<smiles>c1ccc2c3c([nH]c2c1)CCCC3</smiles>

Following the general procedure using cyclohexanone ( $260 \mu \mathrm{L}, 2.5 \mathrm{mmol})$ and phenylhydrazine $1 \mathrm{a}(270 \mu \mathrm{L}, 2.75 \mathrm{mmol})$ for $1 \mathrm{~h}, 2 \mathrm{a}$ was isolated as a pale yellowish solid (415 $\mathrm{mg}, 97 \%$ ) whose data proved consistent with the literature. ${ }^{1}$

Mp: $115-119{ }^{\circ} \mathrm{C}$ (lit. ${ }^{\text {1a }} 119-120{ }^{\circ} \mathrm{C}$ ); ${ }^{1} \mathrm{H}$ NMR $\left(\mathrm{CDCl}_{3}, 400 \mathrm{MHz}\right): \delta$ 1.85-1.98 (m, 4H), 2.70-2.76 (m, 4H), 7.06-7.16 (m, 2H), $7.28(\mathrm{br} \mathrm{d}, J=7.5 \mathrm{~Hz}, 1 \mathrm{H}), 7.48$ (br d, $J=7.5 \mathrm{~Hz}, 1 \mathrm{H}) 7.63(\mathrm{br} \mathrm{s}, 1 \mathrm{H})$; ${ }^{13} \mathrm{C} \mathrm{NMR}\left(\mathrm{CDCl}_{3}, 100 \mathrm{MHz}\right): \delta 21.1,23.36,23.40,23.44,110.3,110.4,117.9,119.2,121.1$, $128.0,134.2,135.8$

\section{6-Isopropyl-2,3,4,9-tetrahydro-1H-carbazole (2b)}

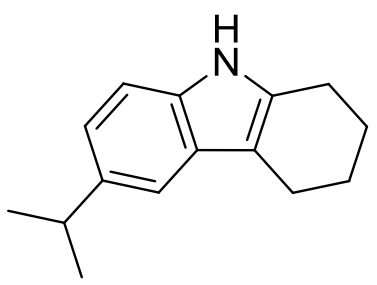

Following the general procedure using cyclohexanone $(230 \mu \mathrm{L}, 2.2$ mmol) and $p$-isopropylphenylhydrazine hydrochloride salt $\mathbf{1} \mathbf{b} \cdot \mathbf{H C l}$ (467 $\mathrm{mg}, 2.4 \mathrm{mmol}$ ) for $1.5 \mathrm{~h}, \mathbf{2 b}$ was isolated as a pale yellowish solid (445 mg, 95\%) whose data proved consistent with the literature. $^{2}$

Mp: 67-69 ${ }^{\circ} \mathrm{C}$ (lit. $\left.{ }^{2} 68-70{ }^{\circ} \mathrm{C}\right) ;{ }^{1} \mathrm{H}$ NMR $\left(\mathrm{CDCl}_{3}, 400 \mathrm{MHz}\right): \delta 1.34$ (d, $J=$ $7.0 \mathrm{~Hz}, 6 \mathrm{H}), 1.87-1.98(\mathrm{~m}, 4 \mathrm{H}), 2.69-2.77(\mathrm{~m}, 4 \mathrm{H}), 3.04$ (hep, $J=7.0 \mathrm{~Hz}, 1 \mathrm{H}), 7.04(\mathrm{dd}, J=8.5$, $2.0 \mathrm{~Hz}, 1 \mathrm{H}), 7.20(\mathrm{~d}, J=8.5 \mathrm{~Hz}, 1 \mathrm{H}), 7.34(\mathrm{~d}, J=2.0 \mathrm{~Hz}, 1 \mathrm{H}), 7.53(\mathrm{br} \mathrm{s}, 1 \mathrm{H}) ;{ }^{13} \mathrm{C} \mathrm{NMR}\left(\mathrm{CDCl}_{3}, 100\right.$ $\mathrm{MHz}): \delta 21.1,23.39,23.40,23.5,24.9,34.4,110.0,110.2,114.8,120.1,128.0,134.3,134.4$, 140.0

\footnotetext{
${ }^{1}$ a) Welch W. M., Synthesis, 1977, 9, 645-646 b) Sun K., Liu S., Bec P. M., and Driver T. G., Ang. Chem. Int. Ed. 2011, 50, 1702-1706

${ }^{2}$ Yeung C. S., Ziegler R. E., Porco J. A., Jr. and Jacobsen E. N., J. Am. Chem. Soc., 2014, 136, 13614-13617
} 


\section{6-Methoxy-2,3,4,9-tetrahydro-1H-carbazole (2c)}<smiles>COc1ccc2[nH]c3c(c2c1)CCCC3</smiles>

Following the general procedure using cyclohexanone $(230 \mu \mathrm{L}, 2.2$ $\mathrm{mmol}$ ) and $p$-methoxyphenylhydrazine hydrochloride salt $\mathbf{1 c} \cdot \mathbf{H C l}$ (442 $\mathrm{mg}, 2.5 \mathrm{mmol}$ ) for $2 \mathrm{~h}, \mathbf{2 c}$ was isolated as a pale pink solid (389 $\mathrm{mg}, 93 \%)$ whose data proved consistent with the literature. ${ }^{3 a}$

Mp: 89-90 ${ }^{\circ} \mathrm{C}$ (lit. 91-92 $\left.{ }^{\circ} \mathrm{C}\right) ;{ }^{1} \mathrm{H}$ NMR $\left(\mathrm{CDCl}_{3}, 400 \mathrm{MHz}\right): \delta$ 1.83-1.95 $(\mathrm{m}, 4 \mathrm{H}), 2.65-2.75(\mathrm{~m}, 4 \mathrm{H}), 3.87(\mathrm{~s}, 3 \mathrm{H}), 6.78(\mathrm{dd}, J=8.5,2.5 \mathrm{~Hz}, 1 \mathrm{H}), 6.94(\mathrm{~d}, J=2.5 \mathrm{~Hz}, 1 \mathrm{H})$, $7.16(\mathrm{~d}, J=8.5 \mathrm{~Hz}, 1 \mathrm{H}$ ), 7.56 (br s, $1 \mathrm{H}) ;{ }^{13} \mathrm{C} \mathrm{NMR}\left(\mathrm{CDCl}_{3}, 100 \mathrm{MHz}\right): \delta 21.1,23.3,23.4,23.5,56.1$, $100.4,110.1,110.6,111.1,128.3,130.9,135.2,154.0$

\section{6-Fluoro-2,3,4,9-tetrahydro-1H-carbazole (2d)}

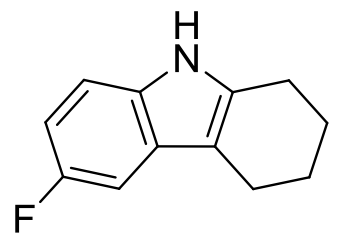

Following the general procedure using cyclohexanone $(230 \mu \mathrm{L}, 2.2$ $\mathrm{mmol}$ ) and $p$-fluorophenylhydrazine hydrochloride salt $\mathbf{1} \mathbf{d} \cdot \mathbf{H C l}(408 \mathrm{mg}$, $2.5 \mathrm{mmol}$ ) for $2 \mathrm{~h}, \mathbf{2} \mathbf{d}$ was isolated as a pale yellowish solid (389 $\mathrm{mg}$, 93\%) whose data proved consistent with the literature. ${ }^{3}$

Mp: $103-104{ }^{\circ} \mathrm{C}$ (lit. $\left.{ }^{3} 106-108{ }^{\circ} \mathrm{C}\right) ;{ }^{1} \mathrm{H}$ NMR $\left(\mathrm{CDCl}_{3}, 400 \mathrm{MHz}\right): \delta 1.83-$ $1.96(\mathrm{~m}, 4 \mathrm{H}), 2.64-2.74(\mathrm{~m}, 4 \mathrm{H}), 6.85(\mathrm{td}, J=9.5,2.5 \mathrm{~Hz}, 1 \mathrm{H}), 7.10(\mathrm{dd}, J=9.5,2.5 \mathrm{~Hz}, 1 \mathrm{H}), 7.17$ (dd, $J=9.0,4.5 \mathrm{~Hz}, 1 \mathrm{H}), 7.65$ (br s, $1 \mathrm{H}) ;{ }^{13} \mathrm{C} \mathrm{NMR}\left(\mathrm{CDCl}_{3}, 100 \mathrm{MHz}\right): \delta 21.0,23.2,23.3,23.4$, $103.0(\mathrm{~d}, J=23.5 \mathrm{~Hz}), 108.9$ (d, $J=26.0 \mathrm{~Hz}), 110.6,110.8(\mathrm{~d}, J=9.5 \mathrm{~Hz}), 128.3,132.2,136.3$, $157.9(d, J=232 \mathrm{~Hz})$

\section{6-Trifluoromethyl-2,3,4,9-tetrahydro-1H-carbazole (2e)}<smiles>FC(F)(F)c1ccc2[nH]c3c(c2c1)CCCC3</smiles>

Following the general procedure using cyclohexanone $(104 \mu \mathrm{L}, 1$ mmol), $p$-trifluoromethylphenylhydrazine $1 \mathrm{e}(1.05 \mathrm{~g}, 6.0 \mathrm{mmol})$ and Amberlite IR $120 \mathrm{H}(2.7 \mathrm{~g})$ for $3 \mathrm{~h}$, filtration on silica with $10 \%$ EtOAc /hexane prior to crystallization affords $\mathbf{2 e}$ as brown yellow solid (220 $\mathrm{mg}, 92 \%)$ whose data proved consistent with the literature. ${ }^{4}$

${ }^{1} \mathrm{H} \mathrm{NMR}\left(\mathrm{CDCl}_{3}, 400 \mathrm{MHz}\right): \delta$ 1.85-1.98 (m, 4H), 2.70-2.77 (m, 4H), 7.30-7.38 (m, 2H), $7.75(\mathrm{~s}$,

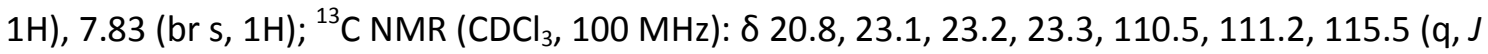
$=4.2 \mathrm{~Hz}), 117.9(\mathrm{q}, J=3.6 \mathrm{~Hz}$ ), $121.6(q, J=31.4 \mathrm{~Hz}), 125.7$ (q, $J=269.6 \mathrm{~Hz}), 127.4,136.1,137.1$

\footnotetext{
${ }^{3}$ a) Chen J. and Hu Y., Synth. Comm. 2006, 36, 1485 b) Sun K., Liu S., Bec P. M., and Driver T. G., Ang. Chem. Int. Ed. 2011, 50, 1702-1706

${ }^{4}$ Desroses M., Wieckowski K., Stevens M. and Odell L. R., Tetrahedron Lett. 2011, 52, 4417-4420
} 


\section{General procedure B:}<smiles>[R]c1ccc(NNN)cc1</smiles>

A stirring solution of phenylhydrazine (10 equiv) and solid acid catalyst Amberlite IR $120 \mathrm{H}^{\circledR}(10$ equiv w/w with respect to 3 ) in $\mathrm{MeOH}(0.05-0.1 \mathrm{M})$ was mixed for $5 \mathrm{~min}$ at $70{ }^{\circ} \mathrm{C}$. To this mixture was added ketone 3 (1 equiv). The mixture was left stirring at $70{ }^{\circ} \mathrm{C}$ for $24 \mathrm{~h}$. After cooling, the reaction mixture was filtered and the resin was washed with $\mathrm{CH}_{2} \mathrm{Cl}_{2}$ and $\mathrm{MeOH}$ and the crude product was purified by crystallization from cold $\mathrm{MeOH}$ or cold $\mathrm{CH}_{2} \mathrm{Cl}_{2}$ to afford the pure carbazole product.

(4aRS,6RS,11bRS)-6-Methyl-4-(4-methylphenylsulfonyl)-2,3,4,4a,5,6,11,11b-octahydro-1Hpyrido[3,2-a]carbazole (4a)

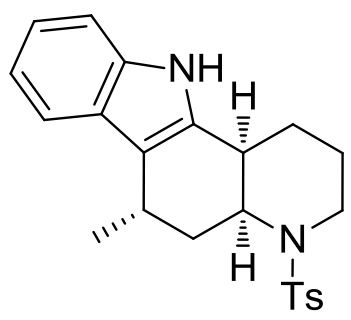

Following the general procedure B using phenylhydrazine 1a $(915 \mu \mathrm{L}$, $9.33 \mathrm{mmol}$ ), 5-oxodecahydroquinoline $3(300 \mathrm{mg}, 0.93 \mathrm{mmol}$ ) and Amberlite IR $120 \mathrm{H}^{\circledR}(3 \mathrm{~g})$ for $20 \mathrm{~h}, \mathbf{4 a}$ was isolated as a pale yellow solid (320 $\mathrm{mg}, 88 \%)$ further crystallization from $\mathrm{CHCl}_{3}$ and dichloroethane afforded pale yellow crystal.

Ts $\mathrm{Mp}: 213-215^{\circ} \mathrm{C} ;{ }^{1} \mathrm{H}$ NMR (COSY, $\left.\mathrm{CDCl}_{3}, 400 \mathrm{MHz}\right): \delta 1.28-1.38(\mathrm{~m}, 1 \mathrm{H}$, $\mathrm{H}-5 \mathrm{eq}), 1.32(\mathrm{~d}, J=7.2 \mathrm{~Hz}, 3 \mathrm{H}, \mathrm{H}-12), 1.53-1.61(\mathrm{~m}, 2 \mathrm{H}, \mathrm{H}-1$ and $\mathrm{H}-2), 1.65-1.70(\mathrm{~m}, 1 \mathrm{H}, \mathrm{H}-2)$, 1.92-2.01 (m, 1H, H-1), 2.31 (ddd, $J=12.8,12.8,6.0 \mathrm{~Hz}, 1 \mathrm{H}, \mathrm{H}-5 \mathrm{ax}$ ), 2.45 (s, 3H, H-17), 2.88 (ddd, $J=10.4,5.2,5.2 \mathrm{~Hz}, 1 \mathrm{H}, \mathrm{H}-11 \mathrm{~b}$ ), 2.97 (ddd, $J=12.8,12.8,2.8 \mathrm{~Hz}, 1 \mathrm{H}, \mathrm{H}-3 \mathrm{ax}$ ), 3.21 (br quint, $J=7.0 \mathrm{~Hz}, 1 \mathrm{H}, \mathrm{H}-6$ ), 3.95 ( $\mathrm{br} \mathrm{d}, J=13.2 \mathrm{~Hz}, 1 \mathrm{H}, \mathrm{H}-3 \mathrm{eq}$ ), 4.56 (ddd, $J=13.2,5.2,3.2 \mathrm{~Hz}$, $1 \mathrm{H}, \mathrm{H}-4 \mathrm{a}), 7.05-7.15(\mathrm{~m}, 2 \mathrm{H}, \mathrm{H}-8$ and H-9), 7.27 (d, J=8.0 Hz, 1H, H-10), 7.31 (d, J=8.0 Hz, 2H, $\mathrm{H}-15), 7.47$ (d, J = 7.6 Hz, 1H, H-7), 7.66 (br s, $1 \mathrm{H}, \mathrm{H}-11$ ), 7.76 (d, $J=8.4 \mathrm{~Hz}, 2 \mathrm{H}, \mathrm{H}-14$ ); ${ }^{13} \mathrm{C} \mathrm{NMR}$ (100 MHz, HSQC, CDCl $)_{3}$ ): $\delta 21.2$ (C-12), 21.5 (C-17), 24.7 (C-2), 25.8 (C-6), 27.7 (C-1), 28.4 (C-5), 34.2 (C-11b), 40.5 (C-3), 49.1 (C-4a), 110.7 (C-10), 113.5 (C-6a), 118.3 (C-7), 119.3 (C-8), 121.5 (C-9), 126.5 (C-6b), 127.0 (C-14), 129.7 (C-15), 135.8 (C-10a), 136.2 (C-13), 138.5 (C-16), 143.1 (C-11a); HRMS: $\mathrm{m} / \mathrm{z}$ calcd for $\mathrm{C}_{23} \mathrm{H}_{27} \mathrm{~N}_{2} \mathrm{O}_{2} \mathrm{~S}(\mathrm{M}+\mathrm{H})^{+} 395.1788$, found 395.1801

(4aRS,6RS,11bRS)-8-Isopropyl-6-methyl-4-(4-methylphenylsulfonyl)-2,3,4,4a,5,6,11,11boctahydro-1H-pyrido[3,2-a]carbazole (4b)

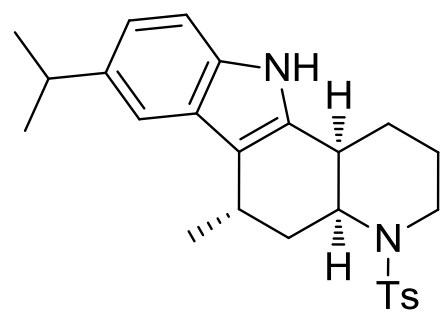

Following the general procedure $\mathrm{B}$ using $p$ isopropylphenylhydrazine $\mathbf{1 b}(467 \mathrm{mg}, 3.11 \mathrm{mmol})$, 5oxodecahydroquinoline 3 (100 $\mathrm{mg}, 0.31 \mathrm{mmol})$ and Amberlite IR $120 \mathrm{H}^{\circledR}(1.00 \mathrm{~g})$ for $20 \mathrm{~h} \mathbf{4 b}$ was isolated after trituration in cold $\mathrm{MeOH}$ and recrystallization in dichloroethane as a white solid (103 mg, 84\%). 
Mp:161-163 ${ }^{\circ} \mathrm{C} ;{ }^{1} \mathrm{H}$ NMR (COSY, $\left.\mathrm{CDCl}_{3}, 400 \mathrm{MHz}\right): \delta 1.25-1.35$ (m, $\left.1 \mathrm{H}, \mathrm{H}-5 \mathrm{eq}\right), 1.30$ (br d, J = 6.8 $\left.\mathrm{Hz}, 6 \mathrm{H}, 2 \times \mathrm{CH}_{3} i \mathrm{Pr}\right), 1.33(\mathrm{~d}, J=7.2 \mathrm{~Hz}, 3 \mathrm{H}, \mathrm{H}-12), 1.52-1.59(\mathrm{~m}, 2 \mathrm{H}, \mathrm{H}-1$ and $\mathrm{H}-2), 1.62-1.68(\mathrm{~m}$, $1 \mathrm{H}, \mathrm{H}-2$ ), 1.92-1.98 (m, $1 \mathrm{H}, \mathrm{H}-1), 2.30$ (ddd, J = 13.2, 13.2, $6.4 \mathrm{~Hz}, 1 \mathrm{H}, \mathrm{H}-5 \mathrm{ax}), 2.45$ (s, 3H, H-17), 2.85 (ddd, $J=11.6,5.0,5.0 \mathrm{~Hz}, 1 \mathrm{H}, \mathrm{H}-11 \mathrm{~b}$ ), 2.91-3.05 (m, 2H, H-3ax \& CH iPr), 3.20 (br quint, $J=$ $7.2 \mathrm{~Hz}, 1 \mathrm{H}, \mathrm{H}-6$ ), 3.94 (br d, J=12.4 Hz, 1H, H-3eq), 4.56 (ddd, J=13.2, 5.0, 3.2 Hz, 1H, H-4a), $7.02(\mathrm{dd}, J=8.4,2.0 \mathrm{~Hz}, 1 \mathrm{H}, \mathrm{H}-9), 7.20(\mathrm{br} \mathrm{d}, J=8.4 \mathrm{~Hz}, 1 \mathrm{H}, \mathrm{H}-10), 7.27-7.37(\mathrm{~m}, 3 \mathrm{H}, \mathrm{H}-7$ \& H15), 7.56 (br s, $1 \mathrm{H}, \mathrm{H}-11$ ), 7.76 (d, $J=8.4 \mathrm{~Hz}, 2 \mathrm{H}, \mathrm{H}-14) ;{ }^{13} \mathrm{C}$ NMR $\left(100 \mathrm{MHz}, \mathrm{HSQC}, \mathrm{CDCl}_{3}\right): \delta$ 21.2 (C-12), 21.5 (C-17), 24.68 (C-2), 24.75 ( $\mathrm{CH}_{3}$ iPr), 25.8 (C-6), 27.7 (C-1), 28.5 (C-5), 34.25 (CH iPr), 34.28 (C-11b), 40.5 (C-3), 49.1 (C-4a), 110.5 (C-10), 113.3 (C-6a), 115.3 (C-7), 120.5 (C-9), 126.5 (C-6b), 127.0 (C-14), 129.7 (C-15), 134.8 (C-10a), 136.0 (C-8),138.5 (C-13), 140.2 (C-16), 143.0 (C-11a); HRMS: $\mathrm{m} / \mathrm{z}$ calcd for $\mathrm{C}_{26} \mathrm{H}_{33} \mathrm{~N}_{2} \mathrm{O}_{2} \mathrm{~S}(\mathrm{M}+\mathrm{H})^{+} 437.2257$, found 437.2256

(4aRS,6RS,11bRS)-8-Methoxy-6-methyl-4-(4-methylphenylsulfonyl)-2,3,4,4a,5,6,11,11boctahydro-1H-pyrido[3,2-a]carbazole (4c)<smiles>COc1ccc2[nH]c3c(c2c1)[C@H](C)C[C@H]1[C@@H]3CCCN1[13CH3]</smiles>

A stirring solution of $p$-methoxyphenylhydrazine hydrochloride 1c (380 mg, $2.17 \mathrm{mmol})$, and solid acid catalyst Amberlite IR $120 \mathrm{H}^{\circledR}$ (350 mg, 5 equiv w/w with respect to 3 ) in $\mathrm{MeOH}(2.1 \mathrm{~mL}, 0.1 \mathrm{M})$ was mix for 5 minutes at $70{ }^{\circ} \mathrm{C}$. On this mixture was added the 5-oxodecahydroquinoline 3 (70 $\mathrm{mg}, 0.22 \mathrm{mmol}$ ). The mixture was left stirring at $70^{\circ} \mathrm{C}$ for $24 \mathrm{~h}$. After cooling, the reaction mixture was filtered and the resin was washed with $\mathrm{CH}_{2} \mathrm{Cl}_{2}$ and $\mathrm{MeOH}$ and the crude product was purified by chromatography (10-25-50\% EtOAc/hexane) to afford $69 \mathrm{mg}$ of a mixture $4 \mathrm{c} / 7 \mathrm{c}$ in a ratio 33/67 (yield of $4 \mathrm{c}$ : $24 \%$ ). (NMR data of $4 c$ were determined by removing signals of $7 c$ ).

${ }^{1} \mathrm{H}$ NMR (COSY, $\left.\mathrm{CDCl}_{3}, 400 \mathrm{MHz}\right): \delta 1.28-1.35(\mathrm{~m}, 1 \mathrm{H}, \mathrm{H}-5 \mathrm{eq}), 1.31$ (d, J=7.2 Hz, 3H, H-12), 1.50-1.62 (m, $2 \mathrm{H}, \mathrm{H}-1$ and $\mathrm{H}-2)$, 1.62-1.70 (m, $1 \mathrm{H}, \mathrm{H}-2), 1.92-2.00(\mathrm{~m}, 1 \mathrm{H}, \mathrm{H}-1), 2.31$ (ddd, $J=$ $12.8,12.8,6.0 \mathrm{~Hz}, 1 \mathrm{H}, \mathrm{H}-5 \mathrm{ax}), 2.44$ (s, 3H, H-17), 2.80-2.88 (m, 1H, H-11b), 2.92-3.02 (m, 1H, H3ax), 3.12-3.20 (m, 1H, H-6), 3.83 (s, 3H, OMe), 3.95 (br d, J = $12.8 \mathrm{~Hz}, 1 \mathrm{H}, \mathrm{H}-3 \mathrm{eq}), 4.55$ (ddd, J $=13.2,5.2,2.8 \mathrm{~Hz}, 1 \mathrm{H}, \mathrm{H}-4 \mathrm{a}), 6.78(\mathrm{dd}, J=8.4,2.4 \mathrm{~Hz}, 1 \mathrm{H}, \mathrm{H}-9), 6.91(\mathrm{~d}, J=2.4 \mathrm{~Hz}, 1 \mathrm{H}, \mathrm{H}-7)$, $7.16(\mathrm{~d}, J=8.4 \mathrm{~Hz}, 1 \mathrm{H}, \mathrm{H}-10), 7.31$ (d, J = $8.0 \mathrm{~Hz}, 2 \mathrm{H}, \mathrm{H}-15), 7.53$ (br s, $1 \mathrm{H}, \mathrm{H}-11), 7.75$ (d, J = 8.4 $\mathrm{Hz}, 2 \mathrm{H}, \mathrm{H}-14$ ); ${ }^{13} \mathrm{C}$ NMR (100 MHz, HSQC, CDCl $)^{\text {): } \delta} 21.0$ (C-12), 21.5 (C-17), 24.7 (C-2), 25.8 (C6), 27.8 (C-1), 28.5 (C-5), 34.3 (C-11b), 40.5 (C-3), 49.1 (C-4a), 56.0 (OMe), 100.9 (C-7), 111.0 (C9), 111.4 (C-10), 113.4 (C-6a), 126.9 (C-6b), 127.0 (C-14), 129.7 (C-15), 131.3 (C-10a), 136.8 (C13),138.5 (C-16), 143.0 (C-11a), 153.9 (C-8); HRMS: m/z calcd for $\mathrm{C}_{24} \mathrm{H}_{29} \mathrm{~N}_{2} \mathrm{O}_{3} \mathrm{~S}(\mathrm{M}+\mathrm{H})^{+}$ 425.1893, found 425.1881

(4aRS,6RS,11bRS)-8-Fluoro-6-methyl-4-(4-methylphenylsulfonyl)-2,3,4,4a,5,6,11,11boctahydro-1H-pyrido[3,2-a]carbazole (4d)

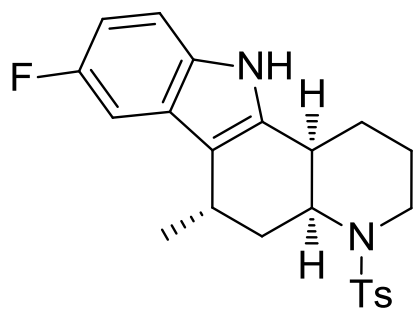

Following the general procedure B using $p$-fluorophenylhydrazine 1d (392 mg, $3.11 \mathrm{mmol}$ ), 5-oxodecahydroquinoline 3 (100 mg, $0.31 \mathrm{mmol})$ and Amberlite IR $120 \mathrm{H}^{\circledR}(1.00 \mathrm{~g})$ for $20 \mathrm{~h}, \mathbf{4 d}$ was isolated after trituration in cold $\mathrm{MeOH}$ and recrystallization in dichloroethane as a white solid (96 mg, 75\%). 
Mp: 242-244 ${ }^{\circ} \mathrm{C} ;{ }^{1} \mathrm{H}$ NMR (COSY, $\mathrm{CDCl}_{3}, 400 \mathrm{MHz}$ ): $\delta$ 1.25-1.35 (m, $1 \mathrm{H}, \mathrm{H}-5$ eq), 1.29 (d, J = 6.8 $\mathrm{Hz}, 3 \mathrm{H}, \mathrm{H}-12), 1.52-1.63(\mathrm{~m}, 2 \mathrm{H}, \mathrm{H}-1$ and $\mathrm{H}-2), 1.63-1.73(\mathrm{~m}, 1 \mathrm{H}, \mathrm{H}-2), 1.92-2.02(\mathrm{~m}, 1 \mathrm{H}, \mathrm{H}-1)$, 2.30 (ddd, $J=12.8,12.8,6.2 \mathrm{~Hz}, 1 \mathrm{H}, \mathrm{H}-5 \mathrm{ax}$ ), 2.45 (s, 3H, H-17), 2.87 (ddd, J = 11.6, 5.0, $5.0 \mathrm{~Hz}$, $1 \mathrm{H}, \mathrm{H}-11 \mathrm{~b}$ ), 2.96 (br t, $J=12.8 \mathrm{~Hz}, 2 \mathrm{H}, \mathrm{H}-3 \mathrm{ax}$ ), 3.14 (br quint, $J=6.8 \mathrm{~Hz}, 1 \mathrm{H}, \mathrm{H}-6), 3.94$ (br d, $J=$ $12.8 \mathrm{~Hz}, 1 \mathrm{H}, \mathrm{H}-3 \mathrm{eq}$ ), 4.54 (ddd, $J=13.2,5.2,3.2 \mathrm{~Hz}, 1 \mathrm{H}, \mathrm{H}-4 \mathrm{a}$ ), 6.86 (ddd, $J=8.8,8.8,2.4, \mathrm{~Hz}$, $1 \mathrm{H}, \mathrm{H}-9$ ), 7.09 (dd, $J=9.0,2.4 \mathrm{~Hz}, 1 \mathrm{H}, \mathrm{H}-7$ ), 7.17 (dd, $J=8.8,4.4 \mathrm{~Hz}, 1 \mathrm{H}, \mathrm{H}-10$ ), 7.31 (d, J = 8.4 $\mathrm{Hz}, 1 \mathrm{H}, \mathrm{H}-15), 7.64$ (br s, 1H, H-11), 7.75 (d, J = 8.4 Hz, 2H, H-14); ${ }^{13} \mathrm{C}$ NMR (100 MHz, HSQC, $\mathrm{CDCl}_{3}$ ): $\delta 21.0$ (C-12), 21.5 (C-17), 24.7 (C-2), 25.7 (C-6), 27.7 (C-1), 28.3 (C-5), 34.3 (C-11b), 40.4 (C-3), 49.0 (C-4a), 103.4 (d, J = 23.3 Hz, C-7), 109.5 (d, J = 26.1 Hz, C-9), 111.2 (d, J = 9.6 Hz, C10), 113.8 (C-6a), 126.9 (C-6b), 127.0 (C-14), 129.7 (C-15), 132.7 (C-10a), 137.8 (C-13), 138.4 (C16), 143.1 (C-11a), 157.7 (d, $J=234.4 \mathrm{~Hz}, \mathrm{C}-8)$; HRMS: $\mathrm{m} / \mathrm{z}$ calcd for $\mathrm{C}_{23} \mathrm{H}_{26} \mathrm{FN}_{2} \mathrm{O}_{2} \mathrm{~S}(\mathrm{M}+\mathrm{H})^{+}$ 413.1694, found 413.1684

(4aRS,6RS,11bRS)-8-Trifluoromethyl-6-methyl-4-(4-methylphenylsulfonyl)2,3,4,4a,5,6,11,11b-octahydro-1H-pyrido[3,2-a]carbazole (4e)<smiles>[3H]N1CCC[C@H]2[C@@H](C)c3c([nH]c4ccc(C(F)(F)F)cc34)[C@@H]2C[C@@H]1C</smiles>

Following the general procedure $\mathrm{B}$ using $p$ trifluoromethylphenylhydrazine 1 e $(548 \mathrm{mg}, 3.11 \mathrm{mmol})$, 5oxodecahydroquinoline 3 (100 $\mathrm{mg}, 0.31 \mathrm{mmol})$ and Amberlite IR $120 H^{\circledR}(1.00 \mathrm{~g})$ for $72 \mathrm{~h}$. Purification by chromatography (10-25-50\% EtOAc/hexane) followed by trituration in cold $\mathrm{MeOH}$ allowed to obtain a mixture $4 \mathrm{e} / 7 \mathrm{e}$ in a ratio $75 / 25$ (yield of $4 \mathrm{e}: 8 \mathrm{mg}, 5.5 \%)$. Another fraction was isolated $19 \mathrm{mg}$ containing $50 \%$ of 4 e/7e $1 / 2$. The remaining part corresponding to hydrazone and 5 oxodecahydroquinoline 3 (yield of $4 \mathrm{e}$ combined: $8 \%$ ). (NMR data of $4 \mathrm{e}$ were determined by removing signals of $7 \mathbf{e})$.

${ }^{1} \mathrm{H}$ NMR (COSY, $\left.\mathrm{CDCl}_{3}, 400 \mathrm{MHz}\right): \delta 1.28-1.35$ (m, 1H, H-5eq), 1.32 (d, J = 6.8 Hz, 3H, H-12), 1.52-1.63 (m, 2H, H-1 \& H-2), 1.66-1.73 (m, 1H, H-2), 1.95-2.05 (m, 1H, H-1), 2.31 (ddd, $J=$ 13.2, 13.2, $6.8 \mathrm{~Hz}, 1 \mathrm{H}, \mathrm{H}-5 \mathrm{ax}), 2.45$ (s, 3H, H-17), 2.89-3.08 (m, 2H, H-3ax \& H-11b), 3.22 (quint, $J=6.8 \mathrm{~Hz}, 1 \mathrm{H}, \mathrm{H}-6), 3.95(\mathrm{br} \mathrm{d}, J=12.4 \mathrm{~Hz}, 1 \mathrm{H}, \mathrm{H}-3 \mathrm{eq}$ ), 4.57 (ddd, $J=13.2,5.2,2.8 \mathrm{~Hz}, 1 \mathrm{H}, \mathrm{H}-$ 4a), 7.28-7.38 (m, 4H, H-7, H-10 \& H-15), 7.70-7.74 (m, 3H, H-9 \& H-14), 7.89 (br s, 1H, H-11); ${ }^{13} \mathrm{C} \mathrm{NMR}\left(100 \mathrm{MHz}, \mathrm{HSQC}, \mathrm{CDCl}_{3}\right.$ ): $\delta 21.3$ (C-12), 21.5 (C-17), 24.7 (C-2), 25.6 (C-6), 27.7 (C-1), 28.2 (C-5), 34.3 (C-11b), 40.4 (C-3), 48.9 (C-4a), 110.9 (C-7), 114.4 (C-6a), 115.9 (d, J = 4.1 Hz, C9), 118.3 (d, J=3.4 Hz, C-10), 121.8 (q, J=31.6 Hz, C-8), 125.3 (q, J = 269.6 Hz, C-18), 125.9 (C6b), 127.0 (C-14), 129.8 (C-15), 137.7 (C-13 \& C-10a), 138.4 (C-16), 143.2 (C-11a); HRMS: m/z calcd for $\mathrm{C}_{24} \mathrm{H}_{26} \mathrm{~F}_{3} \mathrm{~N}_{2} \mathrm{O}_{2} \mathrm{~S}(\mathrm{M}+\mathrm{H})^{+}$463.1662, found 463.1675 


\section{General procedure C:}<smiles>[R]c1ccc(NN)cc1</smiles><smiles>C[C@@H]1CC(=O)[C@@H]2CCCN(C)[C@@H]2C1</smiles>

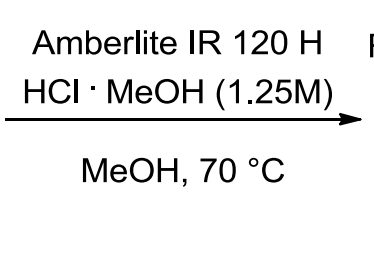<smiles>[R]c1ccc2[nH]c3c(c2c1)[C@H](C)C[C@H]1[C@@H]3CCCN1[Y5]</smiles>

A stirring solution of phenylhydrazine hydrochloride $1 \cdot \mathbf{H C l}$ (2.5 equiv), solid acid catalyst Amberlite IR $120 \mathrm{H}^{\circledR}$ (10 equiv w/w with respect to 3 ) and 5-oxodecahydroquinoline 3 (1 equiv) in $\mathrm{HCl} / \mathrm{MeOH}\left(1.25 \mathrm{M}, 30\right.$ equiv) was stirred at $70{ }^{\circ} \mathrm{C}$ for the indicated time. After cooling, the reaction mixture was filtered and the resin was washed with $\mathrm{CH}_{2} \mathrm{Cl}_{2}$ and $\mathrm{MeOH}$. The excess hydrazine salt was removed by precipitation in cold dichloromethane and the crude product was purified by precipitation from cold $\mathrm{MeOH}$ to afford the pure carbazole product.

(4aRS,6SR,11bRS)-6-Methyl-4-(4-methylphenylsulfonyl)-2,3,4,4a,5,6,11,11b-octahydro-1Hpyrido[3,2-a]carbazole (7a)

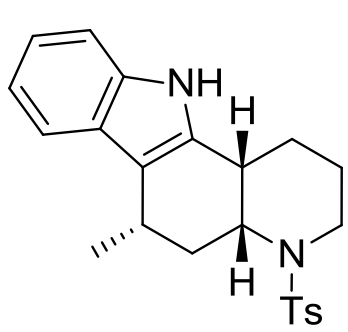

Following the general procedure $\mathrm{C}$ using phenylhydrazine hydrochloride $1 \mathrm{a} \cdot \mathrm{HCl}(61 \mathrm{mg}, 0.42 \mathrm{mmol}), 5$-oxodecahydroquinoline 3 (54 mg, $0.17 \mathrm{mmol}$ ) and Amberlite IR $120 \mathrm{H}^{\circledR}(500 \mathrm{mg}$ ) for $3 \mathrm{~h}, 7 \mathrm{a}$ was isolated as a pale yellow solid (62 $\mathrm{mg}, 92 \%$ ) further crystallization from $\mathrm{CHCl}_{3}$ and DCE afforded pale yellow crystals.

Mp: $205-207{ }^{\circ} \mathrm{C} ;{ }^{1} \mathrm{H}$ NMR (COSY, $\left.\mathrm{CDCl}_{3}, 400 \mathrm{MHz}\right): \delta 1.41(\mathrm{~d}, J=6.8$ $\mathrm{Hz}, 3 \mathrm{H}, \mathrm{H}-12), 1.45-1.52(\mathrm{~m}, 1 \mathrm{H}, \mathrm{H}-2), 1.60-1.72(\mathrm{~m}, 2 \mathrm{H}, \mathrm{H}-1$ \& $\mathrm{H}-2), 1.75-1.93(\mathrm{~m}, 2 \mathrm{H}, \mathrm{H}-5)$, 1.95-2.02 (m, 1H, H-1), 2.44 (s, 3H, H-17), 2.78 (ddd, J = 12.0, 4.8, 4.8 Hz, 1H, H-11b), 3.05 (ddd, $J=13.4,13.4,2.4 \mathrm{~Hz}, 1 \mathrm{H}, \mathrm{H}-3 \mathrm{ax}), 3.10-3.24(\mathrm{~m}, 1 \mathrm{H}, \mathrm{H}-6), 3.92(\mathrm{br} \mathrm{d}, J=13.4 \mathrm{~Hz}, 1 \mathrm{H}, \mathrm{H}-3 e q)$, 4.36 (ddd, $J=12.8,4.8,3.6 \mathrm{~Hz}, 1 \mathrm{H}, \mathrm{H}-4 \mathrm{a}), 7.05-7.15(\mathrm{~m}, 2 \mathrm{H}, \mathrm{H}-8$ \& H-9), 7.27 (d, J = 7.6 Hz, $1 \mathrm{H}$, $\mathrm{H}-10), 7.30$ (d, J = 8.4 Hz, 1H, H-15), 7.61 (d, J = 7.6 Hz,1H, H-7), 7.68 (br s, 1H, H-11), 7.75 (d, J $=8.4 \mathrm{~Hz}, 2 \mathrm{H}, \mathrm{H}-14) ;{ }^{13} \mathrm{C} \mathrm{NMR}\left(100 \mathrm{MHz}, \mathrm{HSQC}, \mathrm{CDCl}_{3}\right.$ ): $\delta 21.3$ (C-12), 21.5 (C-17), 24.5 (C-2), 28.14 (C-1), 28.32 (C-6), 32.3 (C-5), 34.0 (C-11b), 40.5 (C-3), 52.4 (C-4a), 110.7 (C-10), 113.1 (C6a), 119.3 (C-8), 119.9 (C-7), 121.3 (C-9), 126.6 (C-6b), 127.0 (C-14), 129.7 (C-15), 136.1 (C-10a), 136.3 (C-13), 138.5 (C-16), 143.1 (C-11a); HRMS: m/z calcd for $\mathrm{C}_{23} \mathrm{H}_{27} \mathrm{~N}_{2} \mathrm{O}_{2} \mathrm{~S}(\mathrm{M}+\mathrm{H})^{+} 395.1788$, found 395.1805

(4aRS,6SR,11bRS)-8-Isopropyl-6-methyl-4-(4-methylphenylsulfonyl)-2,3,4,4a,5,6,11,11boctahydro-1H-pyrido[3,2-a]carbazole (7b)<smiles>CC(C)c1ccc2[nH]c3c(c2c1)[C@H](C)C[C@@H]1[C@@H]3CCCN1[13CH3]</smiles>

Following the general procedure $\mathrm{C}$ using $p$ isopropylphenylhydrazine hydrochloride $\mathbf{1} \mathbf{b} \cdot \mathbf{H C l}(145 \mathbf{m g}, 0.78$ mmol), 5-oxodecahydroquinoline 3 (100 $\mathrm{mg}, 0.31 \mathrm{mmol}$ ) and Amberlite IR $120 \mathrm{H}^{\oplus}(1.00 \mathrm{~g})$ for $3 \mathrm{~h}, \mathbf{7 b}$ was isolated after trituration in cold $\mathrm{MeOH}$ and recrystallization in DCE as a white solid (119 mg, 88\%). 
${ }^{1} \mathrm{H}$ NMR (COSY, $\mathrm{CDCl}_{3}, 400 \mathrm{MHz}$ ): $\delta 1.29$ (dd, $J=7.5,1.6 \mathrm{~Hz}, 6 \mathrm{H}, 2 \times \mathrm{CH}_{3} i \mathrm{Pr}$ ), 1.40 (d, $J=6.8 \mathrm{~Hz}$, $3 \mathrm{H}, \mathrm{H}-12), 1.43-1.53(\mathrm{~m}, 1 \mathrm{H}, \mathrm{H}-2), 1.53-1.68(\mathrm{~m}, 2 \mathrm{H}, \mathrm{H}-1$ \& $\mathrm{H}-2), 1.72-1.90(\mathrm{~m}, 2 \mathrm{H}, \mathrm{H}-5), 1.91-$ $1.99(\mathrm{~m}, 1 \mathrm{H}, \mathrm{H}-1), 2.42$ (s, 3H, H-17), 2.74 (ddd, $J=12.0,4.8,4.8 \mathrm{~Hz}, 1 \mathrm{H}, \mathrm{H}-11 \mathrm{~b}$ ), 2.91-3.15 (m, $3 \mathrm{H}, \mathrm{CH}$ iPr, H-3ax \& H-6), 3.89 (br d, J = $13.6 \mathrm{~Hz}, 1 \mathrm{H}, \mathrm{H}-3 \mathrm{eq}$ ), 4.32 (ddd, $J=12.8,4.8,3.6 \mathrm{~Hz}, 1 \mathrm{H}$, $\mathrm{H}-4 \mathrm{a}), 7.01(\mathrm{bd}, J=8.4 \mathrm{~Hz} 1 \mathrm{H}, \mathrm{H}-9), 7.18(\mathrm{~d}, J=8,4 \mathrm{~Hz}, 1 \mathrm{H}, \mathrm{H}-10), 7.28$ (d, J= 8.4 Hz, 2H, H-15), 7.43 (bs, $1 \mathrm{H}, \mathrm{H}-7), 7.66$ (s, $1 \mathrm{H}, \mathrm{H}-11), 7.73$ (d, $J=8.4 \mathrm{~Hz}, 2 \mathrm{H}, \mathrm{H}-14) ;{ }^{13} \mathrm{C}$ NMR (100 MHz, HSQC, $\mathrm{CDCl}_{3}$ ): $\delta 21.3$ (C-12), 21.5 (C-17), 24.4 (C-2), $24.7\left(\mathrm{CH}_{3} \mathrm{iPr}\right), 24.8\left(\mathrm{CH}_{3} i \mathrm{Pr}\right), 28.1$ (C-1), 28.3 (C-6), 32.3 (C-5), 34.0 (C-11b), 34. 3 (CH iPr), 40.5 (C-3), 52.5 (C-4a), 110.5 (C-10), 112.7 (C-6a), 116.9 (C-7), 120.3 (C-9), 126.6 (C-6b), 126.8 (C-14), 129.7 (C-15), 134.9 (C-10a), 136.4 (C-8), 138.5 (C13), 139.9 (16), 143.0 (C-11a); HRMS: $\mathrm{m} / \mathrm{z}$ calcd for $\mathrm{C}_{26} \mathrm{H}_{33} \mathrm{~N}_{2} \mathrm{O}_{2} \mathrm{~S}(\mathrm{M}+\mathrm{H})^{+} 437.2257$, found 437.2256

(4aRS,6SR,11bRS)-8-Methoxy-6-methyl-4-(4-methylphenylsulfonyl)-2,3,4,4a,5,6,11,11boctahydro-1H-pyrido[3,2-a]carbazole (7c)<smiles>COc1ccc2[nH]c3c(c2c1)[C@H](C)C[C@H]1[C@H]3CCCN1[13CH3]</smiles>

Following the general procedure $\mathrm{C}$ using $p$ methoxyphenylhydrazine hydrochloride $\mathbf{1 c} \cdot \mathbf{H C l}(136 \mathrm{mg}, 0.78$ $\mathrm{mmol}$ ), 5-oxodecahydroquinoline 3 (100 $\mathrm{mg}, 0.31 \mathrm{mmol}$ ) and Amberlite IR $120 \mathrm{H}^{\circledR}(1.00 \mathrm{~g})$ for $6 \mathrm{~h}, 7 \mathrm{c}$ was isolated after purification by chromatography (10-25-50\% EtOAc/hexane) followed by trituration in cold $\mathrm{MeOH}$ as a light pink solid (75 $\mathrm{mg}, 57 \%)$.

${ }^{1} \mathrm{H}$ NMR (COSY, $\mathrm{CDCl}_{3}, 400 \mathrm{MHz}$ ): $\delta 1.40(\mathrm{~d}, J=6.8 \mathrm{~Hz}, 3 \mathrm{H}, \mathrm{H}-12), 1.45-1.52(\mathrm{~m}, 1 \mathrm{H}, \mathrm{H}-2), 1.60-$ $1.72(\mathrm{~m}, 2 \mathrm{H}, \mathrm{H}-1$ \& H-2), 1.75-1.95 (m, 2H, H-5), 1.95-2.02 (m, 1H, H-1), 2.44 (s, 3H, H-17), 2.74 (ddd, $J=12.0,5.0,5.0 \mathrm{~Hz}, 1 \mathrm{H}, \mathrm{H}-11 \mathrm{~b}$ ), 3.05 (ddd, $J=13.6,13.6,2.4 \mathrm{~Hz}, 1 \mathrm{H}, \mathrm{H}-3 \mathrm{ax}$ ), 3.10-3.20 ( $\mathrm{m}, 1 \mathrm{H}, \mathrm{H}-6), 3.84\left(\mathrm{~s}, 3 \mathrm{H}, \mathrm{CH}_{3} \mathrm{O}\right.$ ), 3.94 (br d, $J=13.6 \mathrm{~Hz}, 1 \mathrm{H}, \mathrm{H}-3$ eq), 4.34 (ddd, $J=12.8,5.0,3.6$ $\mathrm{Hz}, 1 \mathrm{H}, \mathrm{H}-4 \mathrm{a}), 6.79$ (dd, $J=8.8,2.4, \mathrm{~Hz}, 1 \mathrm{H}, \mathrm{H}-9$ ), 7.07 (d, $J=2.4 \mathrm{~Hz}, 1 \mathrm{H}, \mathrm{H}-7$ ), 7.15 (d, $J=8.8$ $\mathrm{Hz}, 1 \mathrm{H}, \mathrm{H}-10), 7.29$ (d, J = 8.4 Hz, 2H, H-15), 7.51 (br s, 1H, H-11), 7.74 (d, J = 8.4 Hz, 2H, H-14); ${ }^{13} \mathrm{C} \mathrm{NMR}\left(100 \mathrm{MHz}, \mathrm{HSQC}, \mathrm{CDCl}_{3}\right.$ ): $\delta 21.2$ (C-12), 21.5 (C-17), 24.4 (C-2), 28.15 (C-1), 28.24 (C-6), 32.3 (C-5), 34.1 (C-11b), 40.5 (C-3), 52.4 (C-4a), $56.1\left(\mathrm{CH}_{3} \mathrm{O}\right), 102.8$ (C-7), 110.8 (C-9), 111.2 (C10), 113.0 (C-6a), 126.96 (C-14), 127.01 (C-6b), 129.7 (C-15), 131.5 (C-10a), 137.1 (C-13), 138.6 (C-16), 143.0 (C-11a), 153.7 (C-8); HRMS: $\mathrm{m} / \mathrm{z}$ calcd for $\mathrm{C}_{24} \mathrm{H}_{29} \mathrm{~N}_{2} \mathrm{O}_{3} \mathrm{~S}(\mathrm{M}+\mathrm{H})^{+} 425.1893$, found 425.1881

\section{(4aRS,6SR,11bRS)-8-Fluoro-6-methyl-4-(4-methylphenylsulfonyl)-2,3,4,4a,5,6,11,11b-} octahydro-1H-pyrido[3,2-a]carbazole (7d)

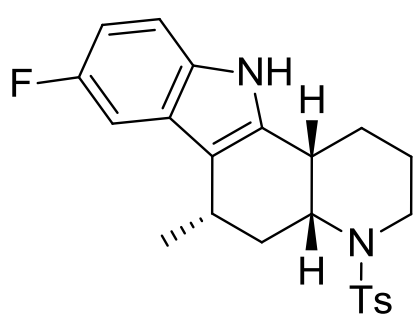

Following the general procedure $\mathrm{C}$ using $p$-fluorophenylhydrazine hydrochloride $\quad \mathbf{1 d} \cdot \mathbf{H C l} \quad(127 \quad \mathrm{mg}, 0.78 \quad \mathrm{mmol}), \quad 5-$ oxodecahydroquinoline 3 (100 $\mathrm{mg}, 0.31 \mathrm{mmol}$ ) and Amberlite IR $120 \mathrm{H}^{\circledR}(1.00 \mathrm{~g})$ for $3 \mathrm{~h}, \mathbf{7 d}$ was isolated after trituration in cold $\mathrm{MeOH}$ and recrystallization in DCE as a white solid (101 $\mathrm{mg}, 82 \%)$.

Ts ${ }^{1} \mathrm{H} \mathrm{NMR}\left(\mathrm{COSY}, \mathrm{CDCl}_{3}, 400 \mathrm{MHz}\right): \delta 1.37(\mathrm{~d}, J=6.4 \mathrm{~Hz}, 3 \mathrm{H}, \mathrm{H}-12)$, 1.46-1.53 (m, $1 \mathrm{H}, \mathrm{H}-2), 1.58-1.72(\mathrm{~m}, 2 \mathrm{H}, \mathrm{H}-1$ \& H-2), 1.73-1.92 (m, 2H, H-5), 1.94-2.03 (m, $1 \mathrm{H}$, $\mathrm{H}-1$ ), 2.44 (s, 3H, H-17), 2.78 (ddd, $J=12.0,5.0,5.0 \mathrm{~Hz}, 1 \mathrm{H}, \mathrm{H}-11 \mathrm{~b}$ ), 3.04 (ddd, $J=13.2,13.2,2.4$ 
$\mathrm{Hz}, 1 \mathrm{H}, \mathrm{H}-3 \mathrm{ax}$ ), 3.08-3.16 (m, 1H, H-6), 3.91 (ddd, J = 13.2, 2.4, $2.4 \mathrm{~Hz}, 1 \mathrm{H}, \mathrm{H}-3 \mathrm{eq}$ ), 4.34 (ddd, $J=$ 12.4, 5.0, $3.6 \mathrm{~Hz}, 1 \mathrm{H}, \mathrm{H}-4 \mathrm{a}$ ), 6.86 (ddd, J = 9.0, 9.0, $2.6 \mathrm{~Hz}, 1 \mathrm{H}, \mathrm{H}-9$ ), 7.16 (dd, J = 9.0, $4.4 \mathrm{~Hz}, 1 \mathrm{H}$, $\mathrm{H}-10), 7.24$ (dd, J = 9.6, $2.6 \mathrm{~Hz}, 1 \mathrm{H}, \mathrm{H}-7$ ), 7.30 (br d, J = 8.4 Hz,2H, H-15), 7.64 (s, 1H, H-11), 7.74 (br d, $J=8.4 \mathrm{~Hz}, 2 \mathrm{H}, \mathrm{H}-14) ;{ }^{13} \mathrm{C}$ NMR (100 MHz, HSQC, CDCl $): \delta 21.0$ (C-12), 21.5 (C-17), 24.5 (C-2), 28.1 (C-1), 28.2 (C-6), 32.2 (C-5), 34.1 (C-11b), 40.5 (C-3), 52.3 (C-4a), 105.0 (d, J =23.9 Hz, C-7), 109.4 (d, J=26.1 Hz, C-9), 111.1 (d, J=9.9 Hz, C-10), 113.4 (C-6a), 126.9 (C-6b), 127.0 (C14), 129.7 (C-15), 132.8 (C-10a), 138.1 (C-13), 138.5 (C-16), 143.1 (C-11a), 157.5 (d, J = 233.8 $\mathrm{Hz}, \mathrm{C}-8)$; HRMS: $\mathrm{m} / \mathrm{z}$ calcd for $\mathrm{C}_{23} \mathrm{H}_{26} \mathrm{FN}_{2} \mathrm{O}_{2} \mathrm{~S}(\mathrm{M}+\mathrm{H})^{+} 413.1694$, found 413.1695

(4aRS,6SR,11bRS)-8-Trifluoromethyl-6-methyl-4-(4-methylphenylsulfonyl)2,3,4,4a,5,6,11,11b-octahydro-1H-pyrido[3,2-a]carbazole (7e)<smiles>[3H]N1CCC[C@@H]2C[C@H](C)CCC[C@H]21</smiles>

Following the general procedure $\mathrm{C}$ using $p$ trifluoromethylphenylhydrazine hydrochloride $1 \mathrm{e} \cdot \mathrm{HCl}(166 \mathrm{mg}$, $0.78 \mathrm{mmol}$ ), 5-oxodecahydroquinoline 3 (100 $\mathrm{mg}, 0.31 \mathrm{mmol}$ ) and Amberlite IR $120 \mathrm{H}^{\otimes}(1.00 \mathrm{~g})$ for $24 \mathrm{~h}, 7 \mathrm{e}$ was isolated after purification by chromatohraphy (10-25-50\% EtOAc/hexane) followed by trituration in cold $\mathrm{MeOH}$ as a white solid (15 mg, $10.5 \%$ ) and the recovered filtrate $50 \mathrm{mg}$ containing $15 \%$ of the product (Yield of 7e combined : 16\%).

${ }^{1} \mathrm{H}$ NMR (COSY, $\mathrm{CDCl}_{3}, 400 \mathrm{MHz}$ ): $\delta 1.40(\mathrm{~d}, J=6.8 \mathrm{~Hz}, 3 \mathrm{H}, \mathrm{H}-12), 1.47-1.53(\mathrm{~m}, 1 \mathrm{H}, \mathrm{H}-2), 1.62-$ $1.71(m, 2 \mathrm{H}, \mathrm{H}-1$ \& H-2), 1.72-1.92 (m, 2H, H-5), 1.97-2.07 (m, 1H, H-1), $2.44(\mathrm{~s}, 3 \mathrm{H}, \mathrm{H}-17), 2.84$ (ddd, $J=12.0,4.8,4.8 \mathrm{~Hz}, 1 \mathrm{H}, \mathrm{H}-11 \mathrm{~b}$ ), 3.03 (ddd, $J=13.2,13.2,2.4 \mathrm{~Hz}, 1 \mathrm{H}, \mathrm{H}-3 \mathrm{ax}$ ), 3.12 (br quint, $J=6.8 \mathrm{~Hz}, 1 \mathrm{H}, \mathrm{H}-6$ ), $3.90(\mathrm{br} \mathrm{d}, J=13.2 \mathrm{~Hz}, 1 \mathrm{H}, \mathrm{H}-3 \mathrm{eq}$ ), 4.36 (ddd, $J=12.8,4.8,3.6 \mathrm{~Hz}$, $1 \mathrm{H}, \mathrm{H}-4 \mathrm{a}$ ), 7.28-7.38 (m, 4H, H-7, H-10 \& H-15), 7.74 (d, J = 8.4 Hz, 2H, H-14), 7.86 (s, 1H, H-9), 7.96 (s, 1H, H-11); ${ }^{13} \mathrm{C}$ NMR (100 MHz, HSQC, CDCl $)$ ): $\delta 21.2$ (C-12), 21.5 (C-17), 24.5 (C-2), 28.06 (C-1), 28.13 (C-6), 31.9 (C-5), 34.1 (C-11b), 40.5 (C-3), 52.2 (C-4a), 110.8 (C-7), 114.0 (C-6a), 117.3 (d, J = 4.2 Hz, C-9), 118.1 (d, J=3.3 Hz, C-10), 121.6 (q, J=31.6 Hz, C-8), 125.4 (q, J = $269.8 \mathrm{~Hz}, \mathrm{C}-18$ ), 126.0 (C-6b), 126.9 (C-14), 129.8 (C-15), 137.8 (C-10a), 138.0 (C-13), 138.4 (C16), 143.2 (C-11a); HRMS: $\mathrm{m} / \mathrm{z}$ calcd for $\mathrm{C}_{24} \mathrm{H}_{26} \mathrm{~F}_{3} \mathrm{~N}_{2} \mathrm{O}_{2} \mathrm{~S}(\mathrm{M}+\mathrm{H})^{+} 463.1662$, found 463.1669 


\section{Synthesis of indoles in flow}

Cartridge assembly:

Both ends of $10-\mathrm{cm}$ Tefzel ${ }^{\circledR}$ (ETFE) tubing (1/8" OD, $1 / 16$ " ID, a) were blocked with cotton wool, fitted with assembled flat bottom super flangeless fittings + metal ferrules (1/8" OD, P359, IDEX, b) and male nut parts (LT-215, IDEX, c). These connections were mounted onto flat unions (P-703-01, IDEX, d). For the filling of the cartridges, only one end was blocked at first, the cartridge was filled with the catalyst ( 100 mg) employing vacuum suction and after, the other end was blocked to seal the cartridge.

\section{Microreactor setup:}

All gas-tight syringes (5 mL, B-247, FutureChemistry Holding BV) were mounted on syringe pumps (B-230, FutureChemistry Holding BV) and connected to Tefzel ${ }^{\circledR}$ tubing $\left(1 / 16^{\prime \prime}\right.$ OD, 1529, IDEX) via female Luer adapters (P-628, IDEX). Throughout the flow system, all the tubing (Tefzel $^{\circledR} 1 / 16^{\prime \prime}$ OD, 1529, IDEX) was assembled with super flangeless nut connections (P-287, IDEX) and assembled ferrules (P-259, IDEX) in order to achieve leak-free fluid connections. Also, a 5 bar back pressure regulator (B-444, FutureChemistry Holding BV) guaranteed pressurization inside the system before eluting into a collection flask (see Figure 1).
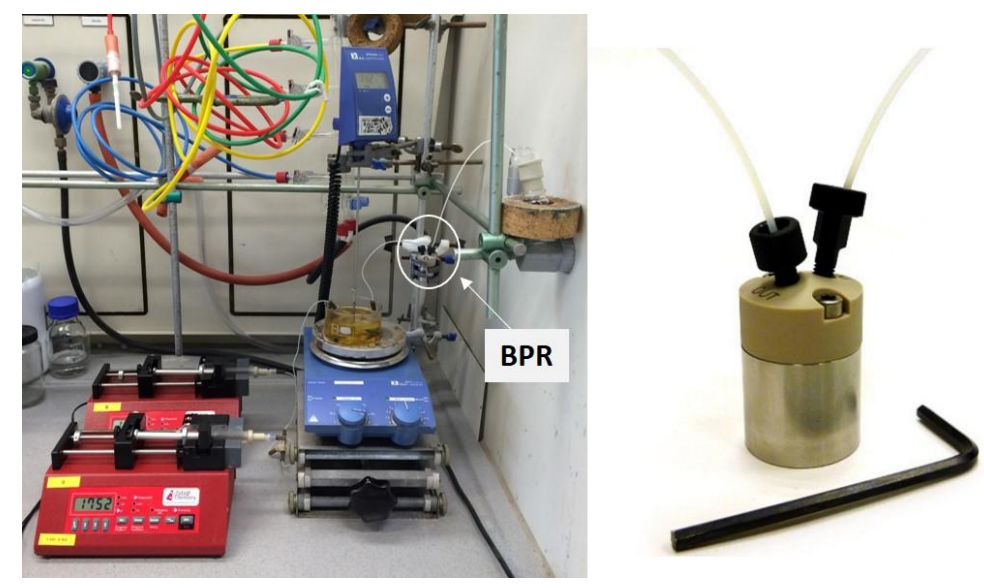

Figure 1. Flow set-up including back pressure regulator (detail, right).

\section{Flow general procedure C:}

Two feed solutions were employed: stream A containing the ketone in solution, and stream B containing the hydrazine in solution both driven by syringe pumps $\left(\phi_{A}=\phi_{B}\right)$. These were mixed in a PEEK T-mixer connection (P-713, IDEX) before entering the microreactor (consisting of a ETFE cartridge packed with Amberlite ${ }^{\circledR} \mathrm{IR} 120 \mathrm{H}$ ) at $70{ }^{\circ} \mathrm{C}$ for 10 to 60 minutes. By removing the solvent in vacuo, the desired indole products were obtained. In some cases, further purification was achieved by recrystallization $\mathrm{CH}_{2} \mathrm{Cl}_{2}$ or methanol.

Full characterization of the indole products can be found within the general procedure for the preparation of the title compounds in batch. 


\section{Calculation for reactions performed under flow conditions:}

For the reactions performed in flow, yields were calculated taking into account the total moles of product obtained ( $n($ Collected Product)), the flow rate ( $\$ S M)$ and the concentration ([SM]) of the starting material and the overall collection time ( $t($ Collection)), as shown in the equation below.

$$
\eta_{\text {Flow }}(\%)=\frac{n(\text { Collected Product })}{[S M] \times \phi_{S M} \times t_{(\text {Collection })}} \times 100
$$

(4aRS,6RS,11bRS)-6-Methyl-4-(4-methylphenylsulfonyl)-2,3,4,4a,5,6,11,11b-octahydro-1Hpyrido[3,2-a]carbazole (4a)<smiles>C[C@H]1C[C@H]2c3[nH]c4ccccc4c3[C@H](CCCN2C)[C@@H]1C(F)(F)F</smiles>

Following the flow general procedure $\mathrm{C}$ using 5oxodecahydroquinoline 3 (0.05 $\mathrm{M}$ in $\mathrm{MeOH} / \mathrm{AcOH} / \mathrm{DCE}$ 4/2/4) and phenylhydrazine 1a $(0.5 \mathrm{M}$ in $\mathrm{MeOH} / \mathrm{AcOH} 1 / 1)$ with reaction time $=$ $20 \mathrm{~min}$, total flow $=15.00 \mu \mathrm{L} \cdot \mathrm{min}^{-1}$ and collecting for $2 \mathrm{~h}(2 \mathrm{~mL}$ of ketone), product 4 a was isolated as a pale yellow solid (31.2 $\mathrm{mg}, 76 \%)$.

(4aRS,6RS,11bRS)-8-Isopropyl-6-methyl-4-(4-methylphenylsulfonyl)-2,3,4,4a,5,6,11,11boctahydro-1H-pyrido[3,2-a]carbazole (4b)<smiles>[3H]N1CCC[C@@H]2c3[nH]c4ccc(C(C)C)cc4c3[C@H](C)C[C@@H]21</smiles>

(32 mg, 75\%).
Following the flow general procedure $\mathrm{C}$ using a $30 \mathrm{~cm}$ cartridge (inner volume $300 \mu \mathrm{L}$ ) 5-oxodecahydroquinoline $3(0.05 \mathrm{M}$ in $\mathrm{MeOH} / \mathrm{AcOH} / \mathrm{DCE}$ 4/2/4) and p-isopropylphenylhydrazine hydrochloride salt $\mathbf{1 b} \cdot \mathbf{H C l}(0.5 \mathrm{M}$ in $\mathrm{MeOH} / \mathrm{AcOH} 1 / 1)$ with reaction time $=30 \mathrm{~min}$, total flow $=10.00 \mu \mathrm{L} \cdot \mathrm{min}^{-1}$ and collecting for $2 \mathrm{~h}$ ( $2 \mathrm{~mL}$ of ketone), the crude ${ }^{1} \mathrm{H}$ NMR spectrum showed full conversion to $\mathbf{4 b}$ which was isolated as a pale yellow solid

(4aRS,6RS,11bRS)-8-Methoxy-6-methyl-4-(4-methylphenylsulfonyl)-2,3,4,4a,5,6,11,11boctahydro-1H-pyrido[3,2-a]carbazole (4c)<smiles>COc1ccc2[nH]c3c(c2c1)[C@H](C)C[C@H]1[C@H]3CCCN1[13CH3]</smiles>

Following the flow general procedure $\mathrm{C}$ using a $1 \mathrm{~m}$ cartridge (inner volume $1 \mathrm{~mL}$ ), 5-oxodecahydroquinoline $3(0.02 \mathrm{M}$ in $\mathrm{MeOH} / \mathrm{AcOH} / \mathrm{DCE}$ 4/4/2) and $p$-methoxyphenylhydrazine hydrochloride salt $\mathbf{1 C} \cdot \mathbf{H C l}(0.2 \mathrm{M}$ in $\mathrm{MeOH})$ with reaction time $=60 \mathrm{~min}$, total flow $=16.00 \mu \mathrm{L} \cdot \mathrm{min}^{-1}$ and collecting for $2 \mathrm{~h}(2$ $\mathrm{mL}$ of ketone), the crude ${ }^{1} \mathrm{H}$ NMR spectrum showed $55 \%$ conversion to $\mathbf{4 c}$ with the remaining part corresponding to a

mix hydrazone/ 5-oxodecahydroquinoline. 
(4aRS,6RS,11bRS)-8-Fluoro-6-methyl-4-(4-methylphenylsulfonyl)-2,3,4,4a,5,6,11,11boctahydro-1H-pyrido[3,2-a]carbazole (4d)<smiles>[3H]N1CCC[C@H]2[C@@H](C)c3c([nH]c4ccc(F)cc34)[C@@H]2C[C@H]1C</smiles>

Following the flow general procedure $\mathrm{C}$ using a $1 \mathrm{~m}$ cartridge (inner volume $1 \mathrm{~mL}$ ), 5-oxodecahydroquinoline $3(0.01 \mathrm{M}$ in $\mathrm{MeOH} / \mathrm{AcOH} / \mathrm{DCE}$ 4/5/1) and p-fluorophenylhydrazine hydrochloride salt $\mathbf{1} \mathbf{d} \cdot \mathbf{H C l}(0.1 \mathrm{M}$ in $\mathrm{MeOH})$ with reaction time $=$ $60 \mathrm{~min}$, total flow $=16.00 \mu \mathrm{L} \cdot \mathrm{min}^{-1}$ and collecting for $2 \mathrm{~h}(2 \mathrm{~mL}$ of

Ts ketone), the crude ${ }^{1} \mathrm{H}$ NMR spectrum showed $42 \%$ conversion to $4 \mathrm{~d}$ with the remaining part corresponding to a mix hydrazone/5-oxodecahydroquinoline.

(4aRS,6RS,11bRS)-8-Trifluoromethyl-6-methyl-4-(4-methylphenylsulfonyl)2,3,4,4a,5,6,11,11b-octahydro-1H-pyrido[3,2-a]carbazole (4e)<smiles>C[C@H]1c2c([nH]c3ccc(C(F)(F)F)cc23)[C@@H]2C[C@@H](C)N([AsH3])CCC[C@@H]12</smiles>

oxodecahydroquinoline.
Following the flow general procedure $\mathrm{C}$ using a $1 \mathrm{~m}$ cartridge (inner volume $1 \mathrm{~mL}$ ), 5-oxodecahydroquinoline $3(0.04 \mathrm{M}$ in $\mathrm{AcOH} / \mathrm{DCE} 8 / 2)$ and $p$-trifluoromethylphenylhydrazine 1e $(0.4$ $\mathrm{M}$ in $\mathrm{MeOH}$ ) with reaction time $=60 \mathrm{~min}$, total flow $=16.00$ $\mu \mathrm{L} \cdot \mathrm{min}^{-1}$ and collecting for $2 \mathrm{~h} \mathrm{(} 2 \mathrm{~mL}$ of ketone), the crude ${ }^{1} \mathrm{H}$ NMR spectrum showed $22 \%$ conversion to $4 \mathrm{e}$ with the remaining part corresponding to a mix hydrazone/ 5- 
Table 1. ${ }^{1} \mathrm{H}$ NMR data of 6-Methyl-4-(4-methylphenylsulfonyl)-1H-2,3,4,4a,5,6,11,11boctahydropyrido[3,2-a]carbazoles

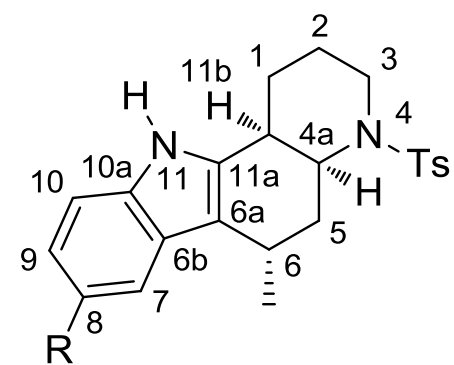

Series 4

\begin{tabular}{|c|c|c|c|c|c|c|}
\hline & $\begin{array}{l}4 a \\
H\end{array}$ & $\begin{array}{l}\mathbf{4 b} \\
\mathrm{Pr}\end{array}$ & $\begin{array}{c}\mathbf{4 c} \\
\mathrm{OMe}\end{array}$ & $\begin{array}{l}\mathbf{4 d} \\
\mathrm{F}\end{array}$ & $\begin{array}{l}\mathbf{4 e} \\
\mathrm{CF}_{3}\end{array}$ \\
\hline \multicolumn{2}{|l|}{$\mathrm{H}-1$} & $\begin{array}{l}1.53-1.61(\mathrm{~m}) \\
1.92-2.01(\mathrm{~m})\end{array}$ & $\begin{array}{l}1.52-1.59(\mathrm{~m}) \\
1.92-1.98(\mathrm{~m})\end{array}$ & $\begin{array}{l}1.50-1.62(\mathrm{~m}) \\
1.92-2.00(\mathrm{~m})\end{array}$ & $\begin{array}{l}1.52-1.63(\mathrm{~m}) \\
1.92-2.02(\mathrm{~m})\end{array}$ & $\begin{array}{l}1.52-1.63(\mathrm{~m}) \\
1.95-2.05(\mathrm{~m})\end{array}$ \\
\hline \multicolumn{2}{|l|}{$\mathrm{H}-2$} & $\begin{array}{l}1.53-1.61(\mathrm{~m}) \\
1.65-1.70(\mathrm{~m})\end{array}$ & $\begin{array}{l}1.52-1,59(\mathrm{~m}) \\
1.62-1.68(\mathrm{~m})\end{array}$ & $\begin{array}{l}1.50-1.62(\mathrm{~m}) \\
1.62-1.70(\mathrm{~m})\end{array}$ & $\begin{array}{l}1.52-1.63(\mathrm{~m}) \\
1-63-1.73(\mathrm{~m})\end{array}$ & $\begin{array}{l}1.52-1.63(\mathrm{~m}) \\
1.66-1.73(\mathrm{~m})\end{array}$ \\
\hline \multirow{2}{*}{$\mathrm{H}-3$} & $a x$ & $\begin{array}{l}2.97 \text { (ddd, 12.8, } \\
12.8,2.8)\end{array}$ & $2.91-3.05(\mathrm{~m})$ & 2.92-3.02 (m) & $2.96(\mathrm{brt}, 12.8)$ & $2.89-3.08(\mathrm{~m})$ \\
\hline & eq & $3.95(\mathrm{br} \mathrm{d}, 13.2)$ & $3.94(\mathrm{br} \mathrm{d}, 12.4)$ & $3.95(\mathrm{br} \mathrm{d}, 12.8)$ & $3.94(\mathrm{br} \mathrm{d}, 12.8)$ & $3.95(\mathrm{br} \mathrm{d}, 12.4)$ \\
\hline \multicolumn{2}{|c|}{$\mathrm{H}-4 \mathrm{a}$} & $\begin{array}{l}4.56 \text { (ddd, 13.2, } \\
5.2,3.2)\end{array}$ & $\begin{array}{l}4.56 \text { (ddd, 13.2, } \\
5.0,3.2)\end{array}$ & $\begin{array}{l}4.55 \text { (ddd, 13.2, } \\
5.2,2.8)\end{array}$ & $\begin{array}{l}4.54 \text { (ddd, 13.2, } \\
5.2,3.2)\end{array}$ & $\begin{array}{l}4.57 \text { (ddd, 13.2, } \\
5.2,2.8)\end{array}$ \\
\hline \multirow{2}{*}{$\mathrm{H}-5$} & $a x$ & $\begin{array}{l}2.31 \text { (ddd, 12.8, } \\
12.8,6.0)\end{array}$ & $\begin{array}{l}2.30 \text { (ddd, 13.2, } \\
13.2,6.4)\end{array}$ & $\begin{array}{l}2.31 \text { (ddd, 12.8, } \\
12.8,6.0)\end{array}$ & $\begin{array}{l}2.30 \text { (ddd, 12.8, } \\
12.8,6.2)\end{array}$ & $\begin{array}{l}2.31 \text { (ddd, 13.2, } \\
13.2,6.8)\end{array}$ \\
\hline & eq & $1.28-1.38(\mathrm{~m})$ & $1.25-1.35(\mathrm{~m})$ & $1.28-1.35(\mathrm{~m})$ & $1.25-1.35(\mathrm{~m})$ & $1.28-1.35(\mathrm{~m})$ \\
\hline \multicolumn{2}{|l|}{$\mathrm{H}-6$} & 3.21 (quint, 7.0 ) & 3.20 (quint, 7.2 ) & $3.12-3.20(\mathrm{~m})$ & 3.14 (quint, 6.8) & 3.22 (quint, 6.8) \\
\hline \multicolumn{2}{|l|}{$\mathrm{H}-7$} & 7.47 & $7.27-7.37$ & 6.91 & 7.09 & $7.28-7.38$ \\
\hline \multicolumn{2}{|l|}{$\mathrm{H}-8$} & $7.05-7.15$ & --- & --- & --- & --- \\
\hline \multicolumn{2}{|l|}{$\mathrm{H}-9$} & $7.05-7.15$ & 7.02 & 6.78 & 6.86 & $7.70-7.74$ \\
\hline \multicolumn{2}{|c|}{$\mathrm{H}-10$} & 7.27 & 7.20 & 7.16 & 7.17 & $7.28-7.38$ \\
\hline \multicolumn{2}{|c|}{$\mathrm{H}-11$} & 7.66 & 7.56 & 7.53 & 7.64 & 7.89 \\
\hline \multicolumn{2}{|c|}{$\mathrm{H}-11 \mathrm{~b}$} & $\begin{array}{l}2.88 \text { (ddd, 11.4, } \\
5.2,5.2)\end{array}$ & $\begin{array}{l}2.85 \text { (ddd, 11.6, } \\
5.0,5.0)\end{array}$ & $2.80-2.88(\mathrm{~m})$ & $\begin{array}{l}2.87 \text { (ddd, 11.6, } \\
5.0,5.0)\end{array}$ & $2.89-3.08(\mathrm{~m})$ \\
\hline \multicolumn{2}{|l|}{$\mathrm{Me}$} & $1.32(\mathrm{~d}, 7.2)$ & 1.33 & 1.31 & 1.29 & 1.32 \\
\hline \multicolumn{2}{|c|}{$\mathrm{H}-14$} & 7.76 & 7.76 & 7.75 & 7.75 & $7.70-7.74$ \\
\hline \multicolumn{2}{|c|}{$\mathrm{H}-15$} & 7.31 & 7.27-7.37 & 7.31 & 7.31 & $7.28-7.38$ \\
\hline \multicolumn{2}{|c|}{ Me-Ts } & 2.45 & 2.45 & 2.44 & 2.45 & 2.45 \\
\hline \multicolumn{2}{|c|}{ Other } & & $\begin{array}{l}1.30 \& 2.91- \\
3.05(\mathrm{Pr})\end{array}$ & $3.84\left(\mathrm{OCH}_{3}\right)$ & & \\
\hline
\end{tabular}




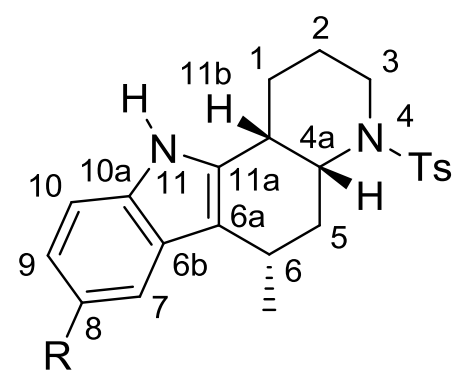

Series 7

\begin{tabular}{|c|c|c|c|c|c|c|}
\hline & $\begin{array}{l}7 a \\
H\end{array}$ & $\begin{array}{l}\text { 7b } \\
\operatorname{Pr}\end{array}$ & $\begin{array}{l}7 \mathrm{c} \\
\mathrm{OMe}\end{array}$ & $\begin{array}{l}7 d \\
F\end{array}$ & $\begin{array}{l}7 e \\
\mathrm{CF}_{3}\end{array}$ \\
\hline \multicolumn{2}{|l|}{$\mathrm{H}-1$} & $\begin{array}{l}1.60-1.72(\mathrm{~m}) \\
1.95-2.02(\mathrm{~m})\end{array}$ & $\begin{array}{l}1.53-1.68(\mathrm{~m}) \\
1.91-1.99(\mathrm{~m})\end{array}$ & $\begin{array}{l}1.60-1.72(\mathrm{~m}) \\
1.95-2.02(\mathrm{~m})\end{array}$ & $\begin{array}{l}1.58-1.72(\mathrm{~m}) \\
1.94-2.03(\mathrm{~m})\end{array}$ & $\begin{array}{l}1.62-1.71(\mathrm{~m}) \\
1.97-2.07(\mathrm{~m})\end{array}$ \\
\hline \multicolumn{2}{|l|}{$\mathrm{H}-2$} & $\begin{array}{l}1.42-1.52(\mathrm{~m}) \\
1.60-1.72(\mathrm{~m})\end{array}$ & $\begin{array}{l}1.43-1.53(\mathrm{~m}) \\
1.53-1.68(\mathrm{~m})\end{array}$ & $\begin{array}{l}1.45-1.52(\mathrm{~m}) \\
1.60-1.72(\mathrm{~m})\end{array}$ & $\begin{array}{l}1.46-1.53(\mathrm{~m}) \\
1.58-1.72(\mathrm{~m})\end{array}$ & $\begin{array}{l}1.47-1.53(\mathrm{~m}) \\
1.62-1.71(\mathrm{~m})\end{array}$ \\
\hline \multirow[t]{2}{*}{$\mathrm{H}-3$} & $a x$ & $\begin{array}{l}3.05 \text { (ddd, 13.4, } \\
13.4,2.4)\end{array}$ & $2.91-3.15(\mathrm{~m})$ & $\begin{array}{l}3.05 \text { (ddd, 13.6, } \\
13.6,2.4)\end{array}$ & $\begin{array}{l}3.04 \text { (ddd, 13.2, } \\
13.2,2.4)\end{array}$ & $\begin{array}{l}3.03 \text { (ddd, 13.2, } \\
13.2,2.4)\end{array}$ \\
\hline & eq & $3.92(\mathrm{br} \mathrm{d}, 13.4)$ & $3.89(\mathrm{br} \mathrm{d}, 13.6)$ & $3.94(\mathrm{br} \mathrm{d}, 13.6)$ & 3.91 (br d, 13.2) & $3.90(\mathrm{br} \mathrm{d}, 13.2)$ \\
\hline \multicolumn{2}{|c|}{$\mathrm{H}-4 \mathrm{a}$} & $\begin{array}{l}4.36(\mathrm{ddd}, 12.8 \\
4.8,3.6)\end{array}$ & $\begin{array}{l}4.32(\mathrm{ddd}, 12.8, \\
4.8,3.6)\end{array}$ & $\begin{array}{l}4.34(\mathrm{ddd}, 12.8 \\
5.0,3.6)\end{array}$ & $\begin{array}{l}4.34 \text { (ddd, 12.4, } \\
5.0,3.6)\end{array}$ & $\begin{array}{l}4.36 \text { (ddd, 12.8, } \\
4.8,3.6)\end{array}$ \\
\hline \multicolumn{2}{|l|}{$\mathrm{H}-5$} & $1.75-1.93(\mathrm{~m})$ & $1.72-1.90(\mathrm{~m})$ & $1.75-1.95(\mathrm{~m})$ & $1.73-1.92(\mathrm{~m})$ & $1.72-1.92(\mathrm{~m})$ \\
\hline \multicolumn{2}{|l|}{$\mathrm{H}-6$} & $3.10-3.24(\mathrm{~m})$ & $2.91-3.15(\mathrm{~m})$ & $3.10-3.20(\mathrm{~m})$ & $3.08-3.16(\mathrm{~m})$ & 3.12 (quint, 6.8) \\
\hline \multicolumn{2}{|l|}{$\mathrm{H}-7$} & 7.61 & 7.43 & 7.07 & 7.24 & $7.28-7.38$ \\
\hline \multicolumn{2}{|l|}{$\mathrm{H}-8$} & $7.05-7.15$ & $\begin{array}{l}-- \\
\end{array}$ & --- & --- & --- \\
\hline \multicolumn{2}{|l|}{$\mathrm{H}-9$} & $7.05-7.15$ & 7.01 & 6.79 & 6.86 & 7.86 \\
\hline \multicolumn{2}{|c|}{$\mathrm{H}-10$} & 7.27 & 7.18 & 7.15 & 7.16 & $7.28-7.38$ \\
\hline \multicolumn{2}{|c|}{$\mathrm{H}-11$} & 7.68 & 7.66 & 7.51 & 7.64 & 7.96 \\
\hline \multicolumn{2}{|c|}{$H-11 b$} & $\begin{array}{l}2.78 \text { (ddd, 12.0, } \\
4.8,4.8)\end{array}$ & $\begin{array}{l}2.74 \text { (ddd, 12.0, } \\
4.8,4.8)\end{array}$ & $\begin{array}{l}2.74 \text { (ddd, 12.0, } \\
5.0,5.0)\end{array}$ & $\begin{array}{l}2.78 \text { (ddd, 12.0, } \\
5.0,5.0)\end{array}$ & $\begin{array}{l}2.84 \text { (ddd, 12.0, } \\
4.8,4.8)\end{array}$ \\
\hline \multicolumn{2}{|l|}{$\mathrm{Me}$} & $1.41(\mathrm{~d}, 6.8)$ & $1.40(\mathrm{~d}, 6.8)$ & $1.40(\mathrm{~d}, 6.8)$ & $1.37(\mathrm{~d}, 6.4)$ & $1.40(\mathrm{~d}, 6.8)$ \\
\hline \multicolumn{2}{|c|}{$\mathrm{H}-14$} & 7.75 & 7.73 & 7.74 & 7.74 & 7.74 \\
\hline \multicolumn{2}{|c|}{$\mathrm{H}-15$} & 7.30 & 7.28 & 7.29 & 7.30 & $7.28-7.38$ \\
\hline \multicolumn{2}{|c|}{ Me-Ts } & 2.44 & 2.42 & 2.44 & 2.44 & 2.44 \\
\hline \multicolumn{2}{|c|}{ Other } & & $\begin{array}{l}1.29 \& \text { \& } 2.91- \\
3.15(\mathrm{IPr})\end{array}$ & $3.84\left(\mathrm{OCH}_{3}\right)$ & & \\
\hline
\end{tabular}


Table 2. ${ }^{13} \mathrm{C}$ NMR data of 6-Methyl-4-(4-methylphenylsulfonyl)-1H-2,3,4,4a,5,6,11,11boctahydropyrido[3,2-a]carbazoles

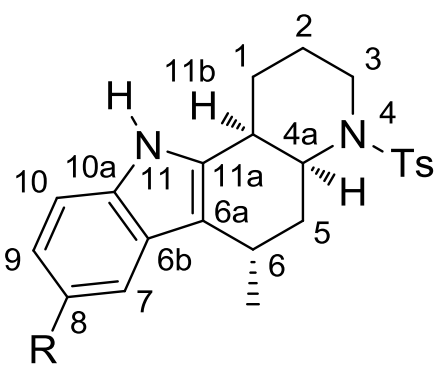

Series 4

\begin{tabular}{|l|l|l|l|l|l|}
\hline & $\begin{array}{l}\mathbf{4} \\
\mathrm{H}\end{array}$ & $\begin{array}{l}\mathbf{4 b} \\
\mathrm{Pr}\end{array}$ & $\begin{array}{l}\mathbf{4 c} \\
\mathrm{OMe}\end{array}$ & $\begin{array}{l}\mathbf{4 d} \\
\mathrm{F}\end{array}$ & $\begin{array}{l}\mathbf{4 e} \\
\mathrm{CF}_{3}\end{array}$ \\
\hline C-1 & 27.7 & 27.7 & 27.8 & 27.7 & 27.7 \\
\hline C-2 & 24.7 & 24.7 & 24.7 & 24.7 & 24.7 \\
\hline C-3 & 40.5 & 40.5 & 40.5 & 40.4 & 40.5 \\
\hline C-4a & 49.1 & 49.1 & 49.1 & 49.0 & 48.9 \\
\hline C-5 & 28.4 & 28.5 & 28.5 & 28.3 & 28.2 \\
\hline C-6 & 25.8 & 25.8 & 25.8 & 25.7 & 25.6 \\
\hline C-6a & 113.5 & 113.3 & 113.4 & 113.8 & 114.4 \\
\hline C-6b & 126.5 & 126.5 & 126.9 & 126.9 & 125.9 \\
\hline C-7 & 118.3 & 115.3 & 100.9 & 103.4 & 110.9 \\
\hline C-8 & 119.3 & 136.0 & 153.9 & 157.7 & 121.6 \\
\hline C-9 & 121.5 & 120.5 & 111.0 & 109.5 & 115.9 \\
\hline C-10 & 110.7 & 110.5 & 111.4 & 111.2 & 118.3 \\
\hline C-10a & 135.8 & 134.8 & 131.3 & 132.7 & 137.7 \\
\hline C-11a & 143.1 & 143.0 & 143.0 & 143.1 & 143.2 \\
\hline C-11b & 34.2 & 34.3 & 34.3 & 34.3 & 34.3 \\
\hline Me & 21.2 & 21.2 & 21.0 & 21.0 & 21.3 \\
\hline Me-Ts & 21.5 & 21.5 & 21.5 & 21.5 & 21.6 \\
\hline C-13 & 136.2 & 138.5 & 136.8 & 137.8 & 137.8 \\
\hline C-14 & 127.0 & 127.0 & 127.0 & 127.0 & 127.0 \\
\hline C-15 & 129.7 & 129.7 & 129.7 & 129.7 & 129.8 \\
\hline C-16 & 138.5 & 140.2 & 138.5 & 138.4 & 138.4 \\
\hline Other & & $24.8 \& 34.3$ & $56.0\left(\mathrm{OCH}_{3}\right)$ & & $125.3\left(\mathrm{CF}_{3}\right)$ \\
\hline & $($ Pr $)$ & & & \\
\hline
\end{tabular}




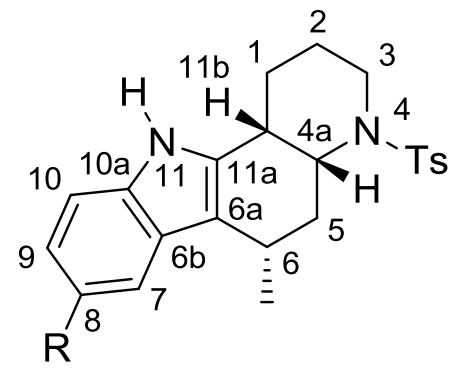

Series 7

\begin{tabular}{|l|l|l|l|l|l|}
\hline & $\begin{array}{l}\mathbf{7 a} \\
\mathrm{H}\end{array}$ & $\begin{array}{l}\mathbf{7 b} \\
\mathrm{Pr}\end{array}$ & $\begin{array}{l}\mathbf{7 c} \\
\mathrm{OMe}\end{array}$ & $\begin{array}{l}\mathbf{7 d} \\
\mathrm{F}\end{array}$ & $\begin{array}{l}\mathbf{7 e} \\
\mathrm{CF}_{3}\end{array}$ \\
\hline $\mathrm{C}-1$ & 28.1 & 28.1 & 28.2 & 28.1 & 28.1 \\
\hline C-2 & 24.5 & 24.4 & 24.4 & 24.5 & 24.5 \\
\hline C-3 & 40.5 & 40.5 & 40.5 & 40.5 & 40.5 \\
\hline C-4a & 52.4 & 52.5 & 52.4 & 52.3 & 52.2 \\
\hline C-5 & 32.3 & 32.3 & 32.3 & 32.2 & 31.9 \\
\hline C-6 & 28.3 & 28.3 & 28.2 & 28.2 & 28.1 \\
\hline C-6a & 113.1 & 112.7 & 113.0 & 113.4 & 114.0 \\
\hline C-6b & 126.6 & 126.6 & 127.01 & 126.9 & 126.0 \\
\hline C-7 & 119.9 & 116.9 & 102.8 & 105.0 & 110.8 \\
\hline C-8 & 119.3 & 136.4 & 153.7 & 157.5 & 121.6 \\
\hline C-9 & 121.3 & 120.3 & 110.8 & 109.4 & 117.3 \\
\hline C-10 & 110.7 & 110.5 & 111.2 & 111.1 & 118.1 \\
\hline C-10a & 136.1 & 134.9 & 131.5 & 132.8 & 137.8 \\
\hline C-11a & 143.1 & 143.0 & 143.0 & 143.1 & 143.2 \\
\hline C-11b & 34.0 & 34.0 & 34.1 & 34.1 & 34.1 \\
\hline Me & 21.3 & 21.3 & 21.2 & 21.0 & 21.2 \\
\hline Me-Ts & 21.5 & 21.5 & 21.5 & 21.5 & 21.5 \\
\hline C-13 & 136.3 & 138.5 & 137.1 & 138.1 & 138.0 \\
\hline C-14 & 127.0 & 126.8 & 126.96 & 127.0 & 126.9 \\
\hline C-15 & 129.7 & 129.7 & 129.7 & 129.7 & 129.8 \\
\hline C-16 & 138.5 & 139.9 & 138.6 & 138.5 & 138.4 \\
\hline Other & & $24.7 \& 34.3$ & $56.1\left(\mathrm{OCH}_{3}\right)$ & & $125.3\left(\mathrm{CF}_{3}\right)$ \\
\hline & $(\mathrm{Pr})$ & & & \\
\hline
\end{tabular}




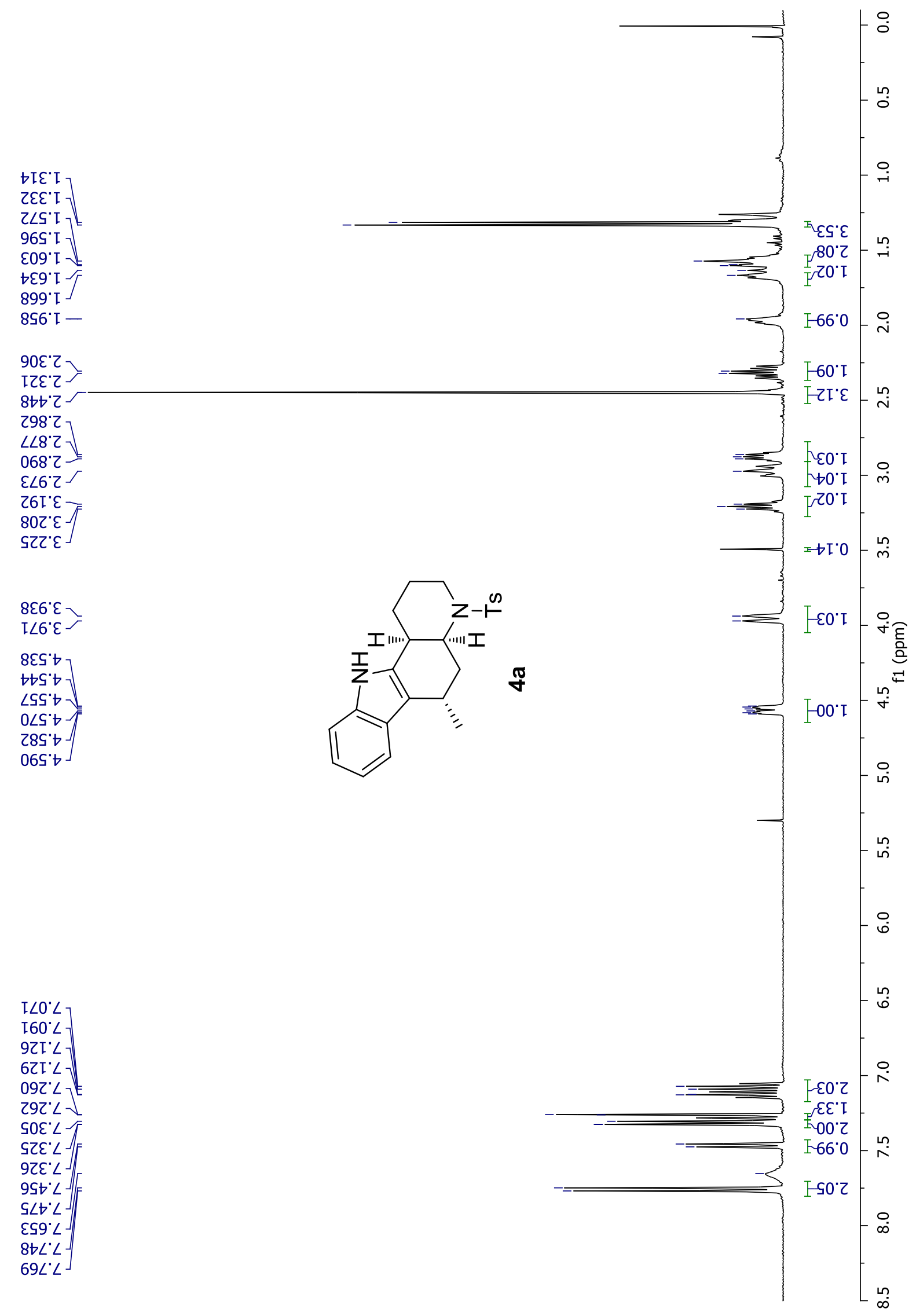


โOZ' IZ

sटS'LC

I08.92 -

$6 \varepsilon L ' L Z-\tau$

$62 b^{\circ} 82$

$8 \varepsilon{ }^{\circ} \sqcup \varepsilon-$

$S \angle t^{\circ} 0 t-$

$\varepsilon 60^{\circ} 6 t-$

$289^{\circ} 9 L$

$000^{\circ} \mathrm{LL}$

$\angle$ IE' $L L$ ]

$9 b \angle ' 0 I T-$

LZS'EI I -

๑62 $8 \mathrm{II}$

$\angle 0 \varepsilon^{\circ} 6$ II

E6t. IZI -

09t.9ZI

966.9ZI $\leftrightharpoons$

†0L'6ZI $\triangle$

96L' ऽEI -

รZZ'9عI

00S'8EI

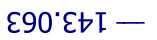

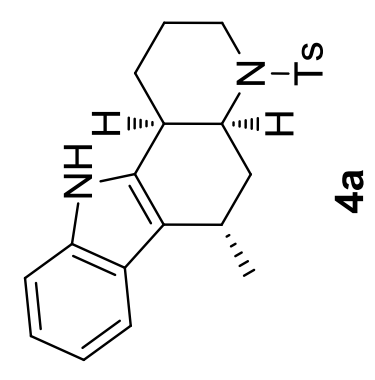

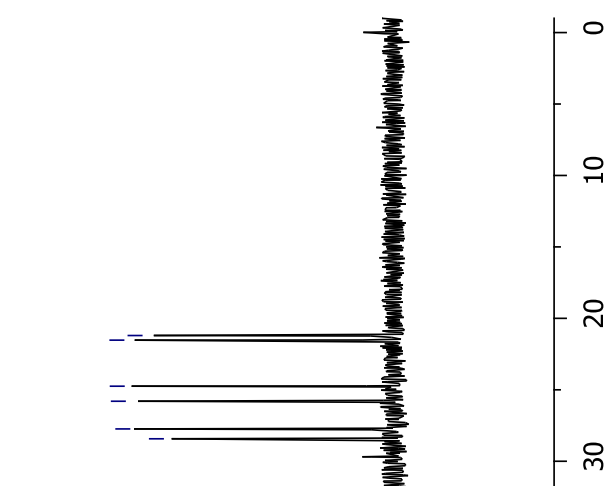

g

in

8

요

틀

요다

8

우

움

స్ా

$\stackrel{\circ}{\oplus}$

g

号 


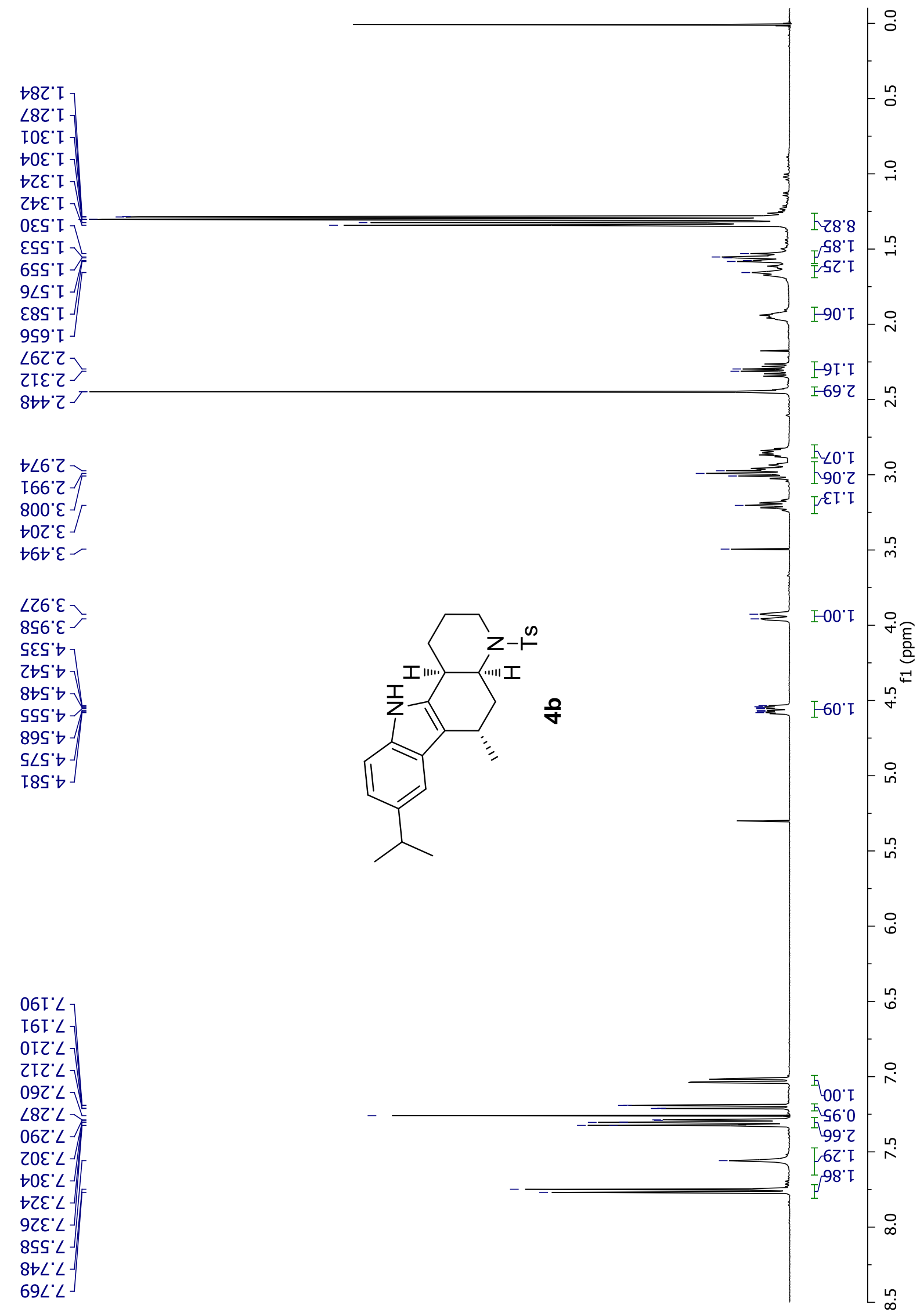




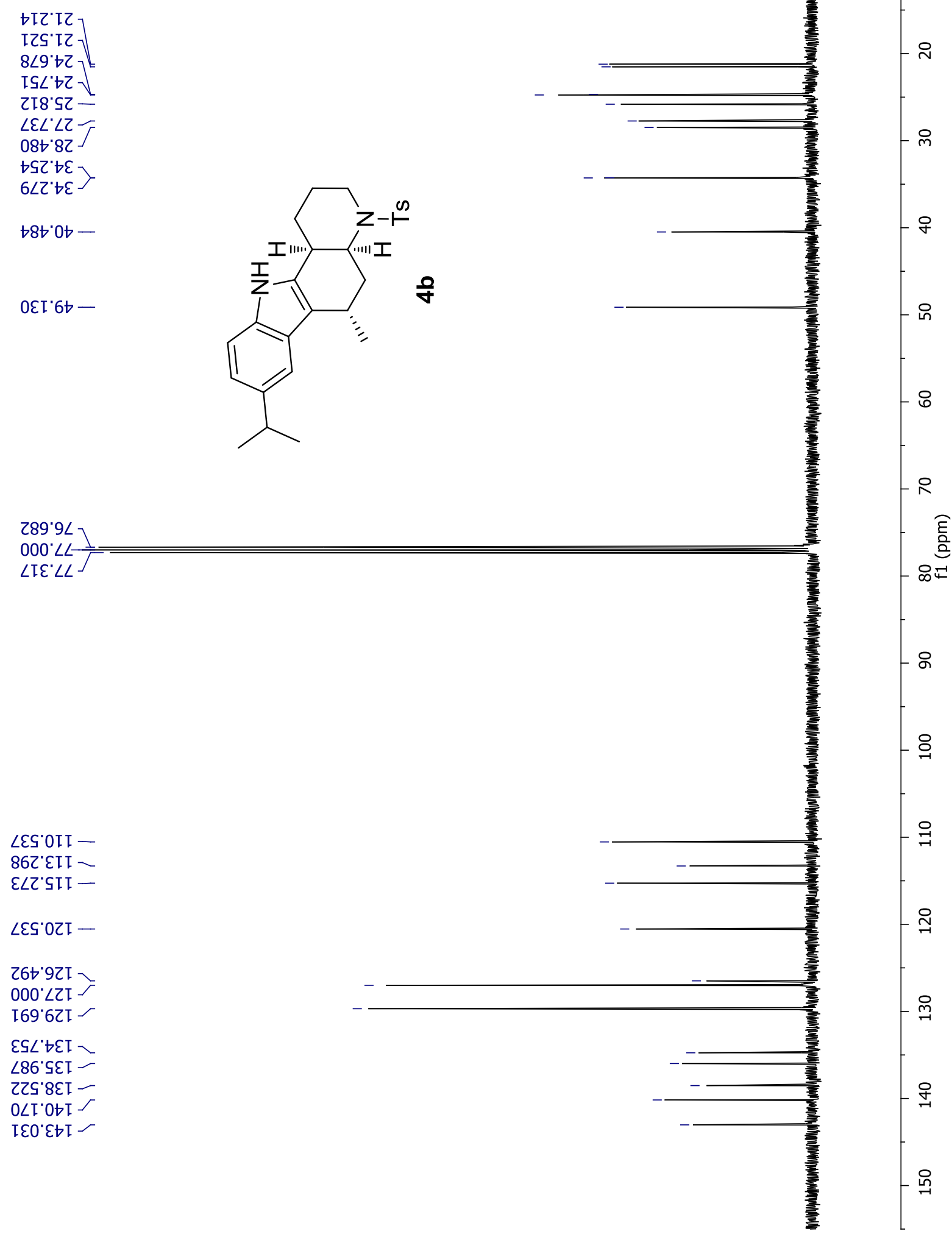




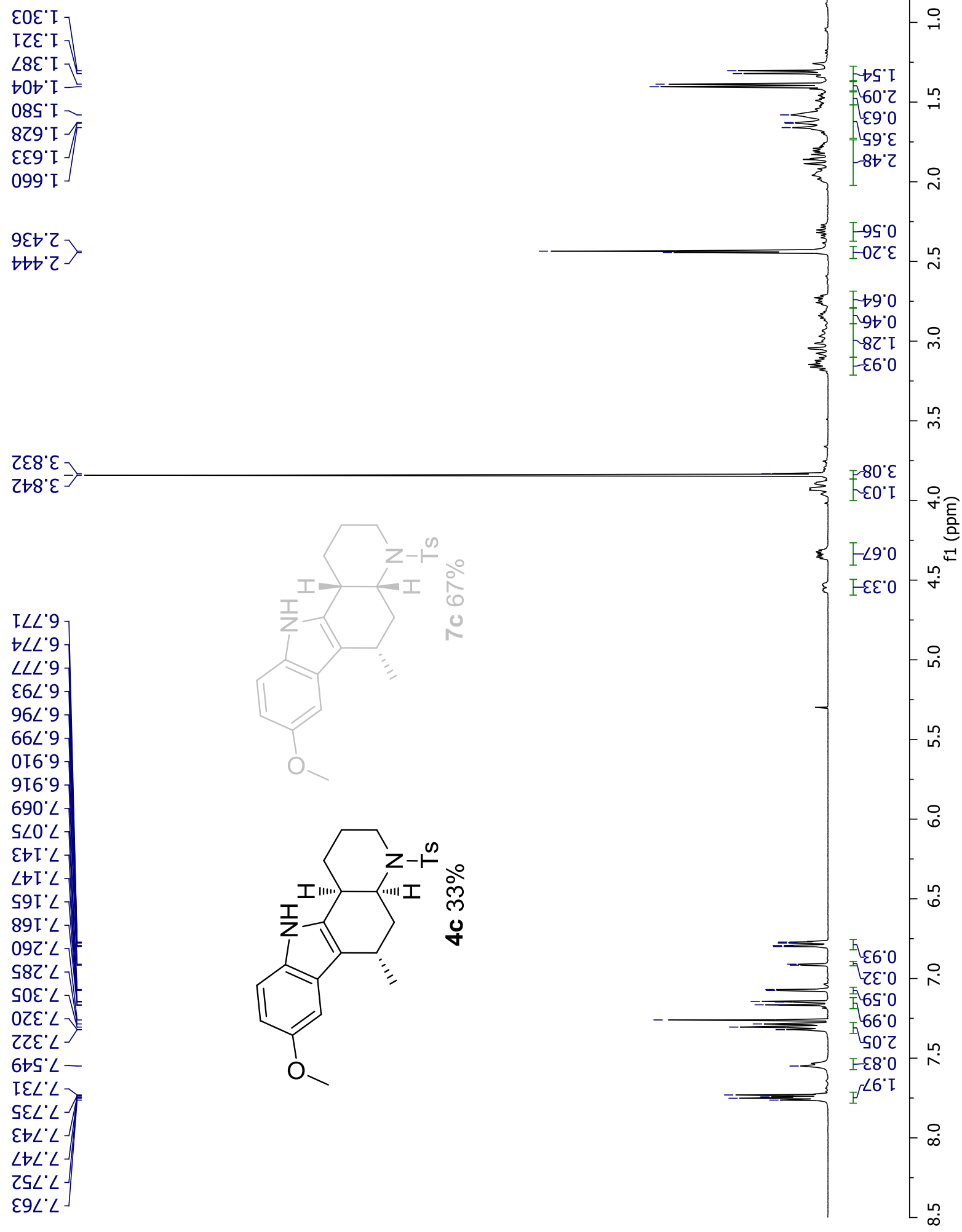




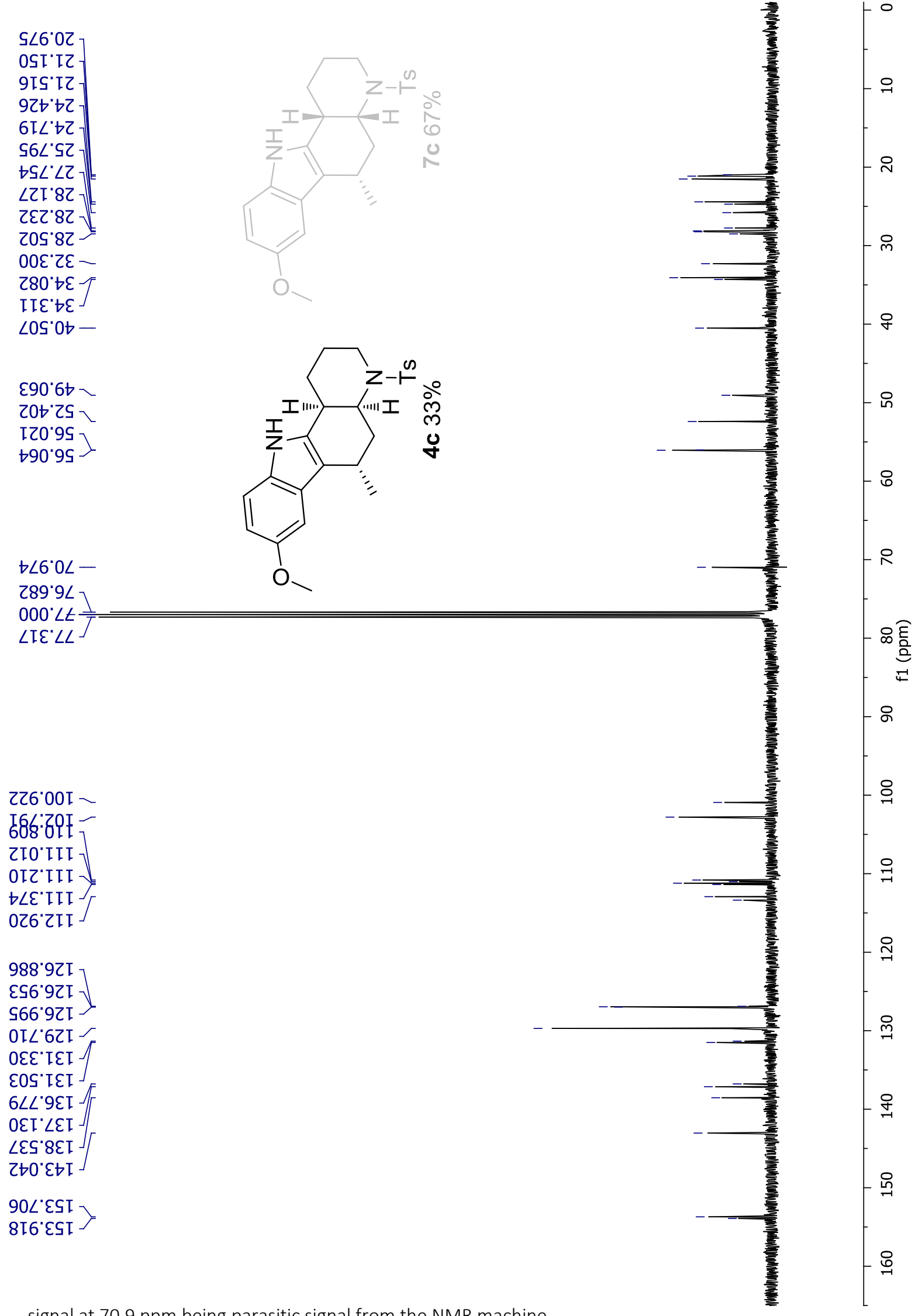

signal at $70.9 \mathrm{ppm}$ being parasitic signal from the NMR machine 
$\angle L Z^{\circ} \cdot$

S62' I

เ9S' I -

$\angle S 6^{\circ} \mathrm{I}$

$896^{\circ} \mathrm{T}$

[86. I

$06 Z^{\prime} Z$

90ع'

$\angle t t^{\circ} 2$

$598^{\circ} \mathrm{Z}$

$088^{\prime} \mathrm{Z}$

$\varepsilon 68$ '

โE6 $2-$

$\angle S 6^{\circ} Z$

乙96'乙

$\angle \varepsilon I^{\prime} \varepsilon$

$0 \varepsilon 6^{\circ} \varepsilon$

$896^{\circ} \varepsilon$

9โS't

EZS' $b]$

$9 \varepsilon S^{\circ}$

$6 t S^{\prime} t-$

9SS $t$

295't -

695 't

$898: 2-$

EOE' $L$

SOE' $\angle]$

ธحE' $\angle$

9२ह $\angle$

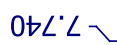

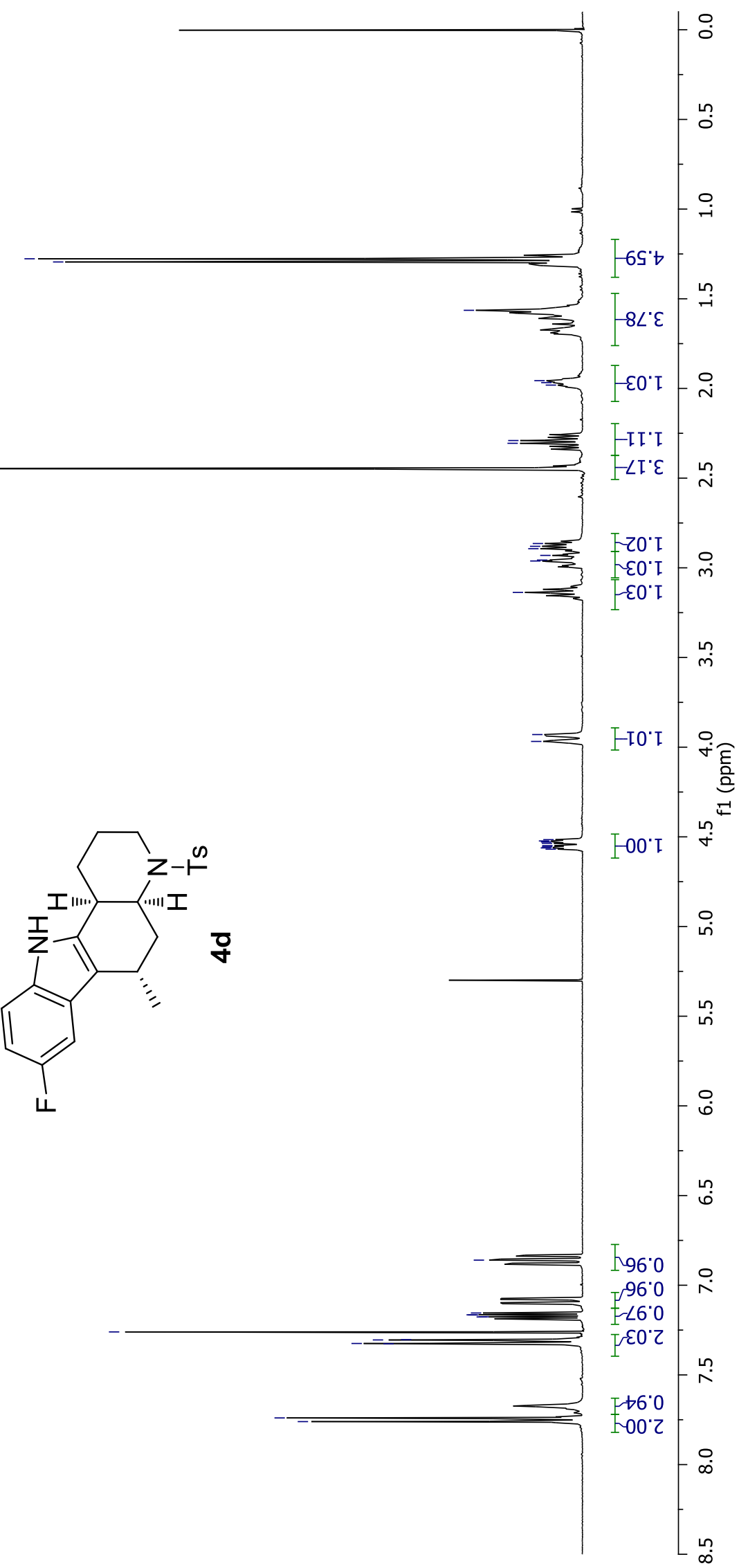


โ66.0Z

E\&S' IZ -

$\left.\varepsilon b L^{\prime} t Z\right\rangle$

IZL'SZ-

I69' $\angle Z-5$

$\angle 8 Z^{\circ} 8 \mathrm{Z}$

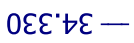

sてtot -

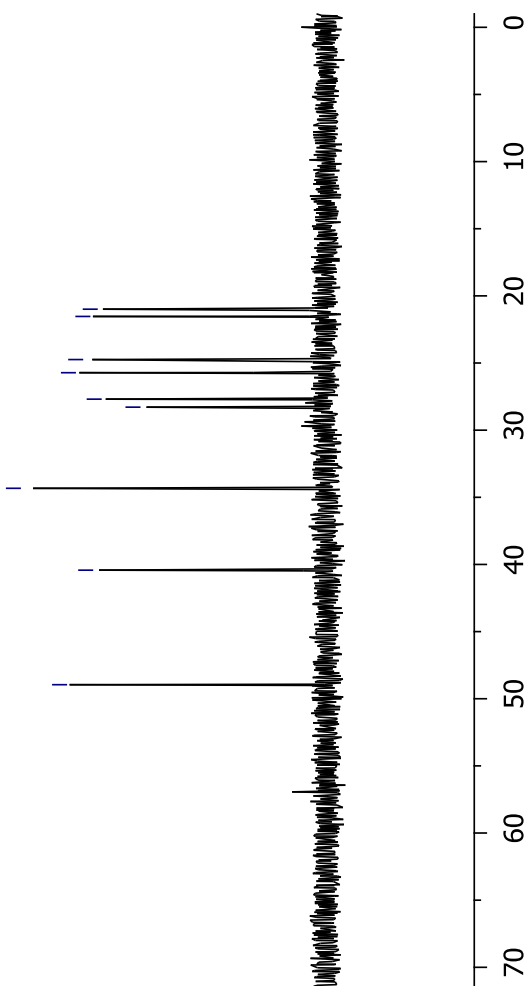

$289^{\circ} 9 L$

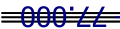

$\angle I E^{\prime} \angle L$

9โ $\varepsilon^{\prime} \varepsilon 0$ I

$\angle \supset \mathcal{S}^{\circ} \varepsilon 0 \mathrm{I}$

$\varepsilon 8 \varepsilon^{\circ} 60[$

$\varepsilon \triangleright 9^{\circ} 60 \mathrm{~T}$

S8I'III-

[8Z' I I I

\&I6.9ZI T

Z66.9ZI

IEL'6ZI

乙८9'Zहा

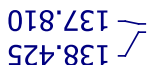

9عโ'E† -

IZS'9SI -

IS8 8 SI -

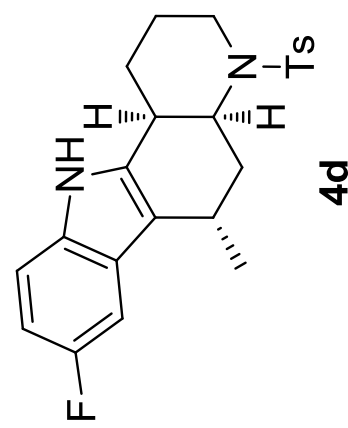




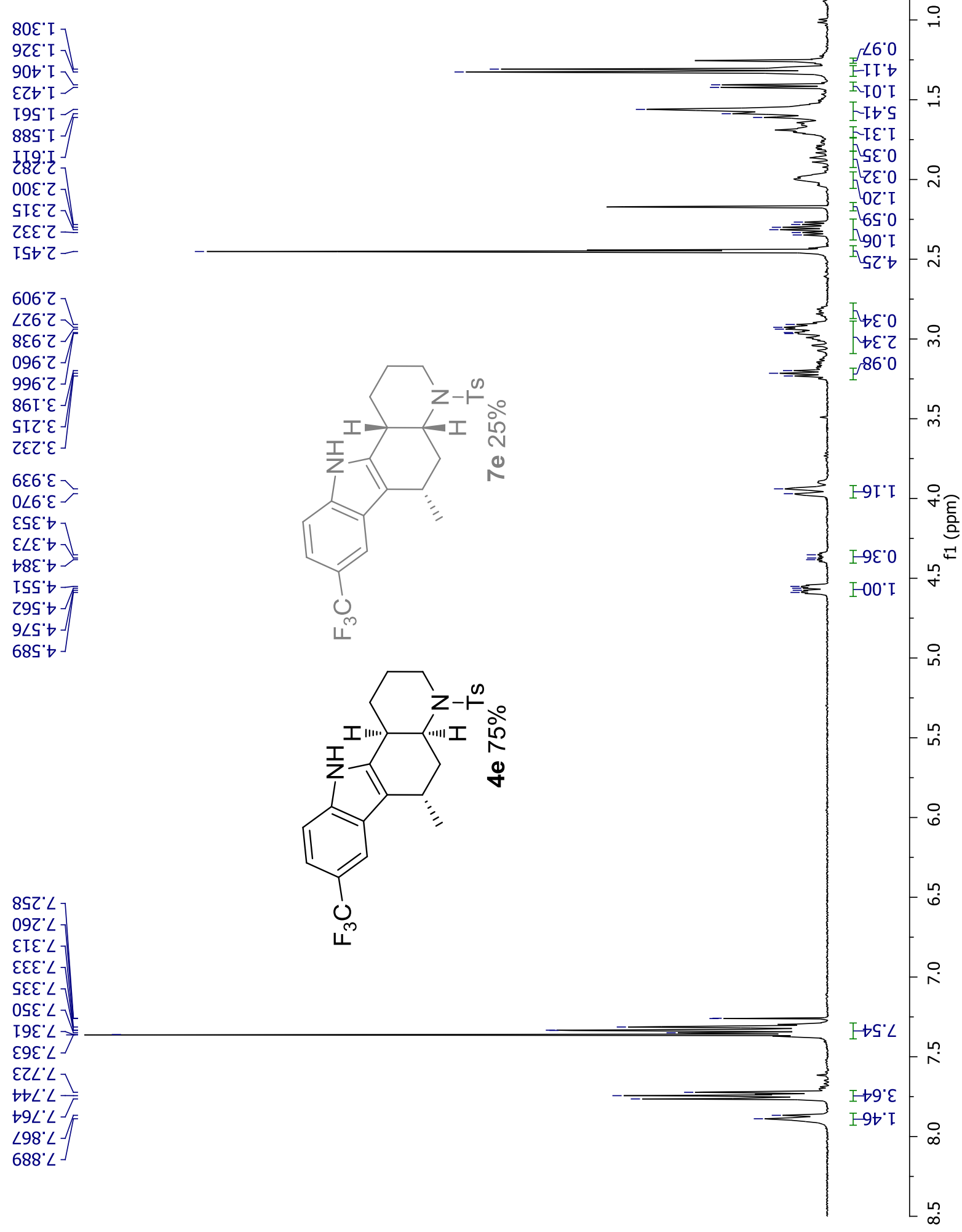


$\angle 0 \varepsilon^{\prime}$ IZ

Z9S'IZ

I9t $b 2$ ]

$09 \angle \circ\rangle$

ह†9's -

$\varepsilon 89^{\circ} \angle Z$

$S \angle 0^{\circ} 8 \mathrm{Z}$

†9 ' $8 \mathrm{C}$ ]

$\angle 0 Z$ ' $8 \mathrm{Z}$

†I $\angle{ }^{\prime} 6 Z$ -

$\angle 20^{\circ} \mathrm{CE} \sim$

$0 \triangleright 0^{\circ} \triangleright \varepsilon-$

692' $\downarrow \varepsilon$

OZt'0t>

08t'0t>

ع88'8t -

LZZ'ZS -

I0L'9L
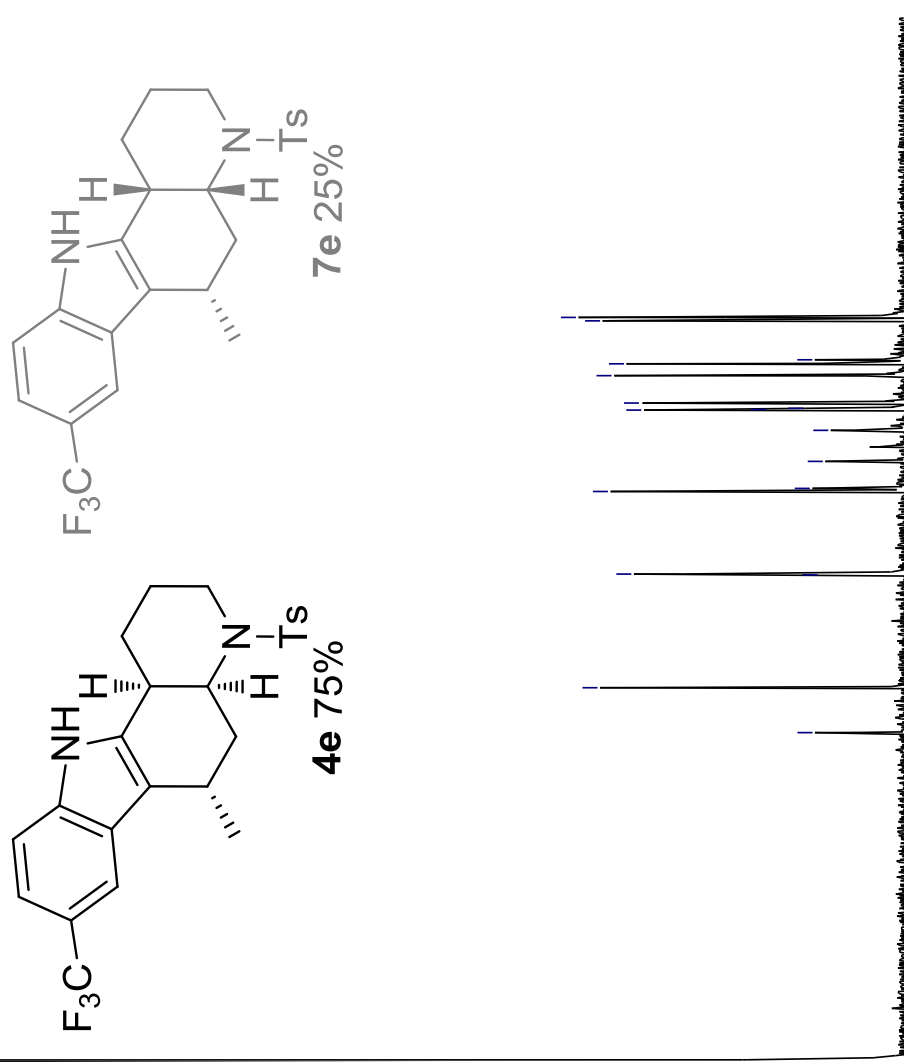

ए

9ع $\angle L$

โE8.0 [ I ]

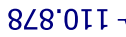

$\varepsilon 9 b^{\circ} \downarrow I I_{]}[$

$\varepsilon \angle 8^{\circ}$ SI I ]

†I6.SII $]$

IEE'8I I T

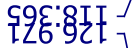

$800^{\circ} \angle Z T$

$0 \rightarrow \varepsilon^{\circ} 8 Z \mathrm{~T}$

98L'6ZI -

0 S9. $\angle E I$

I69. $\angle \varepsilon I$

乙LE $8 \varepsilon[-$

SOt. $8 \varepsilon[$

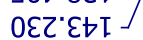

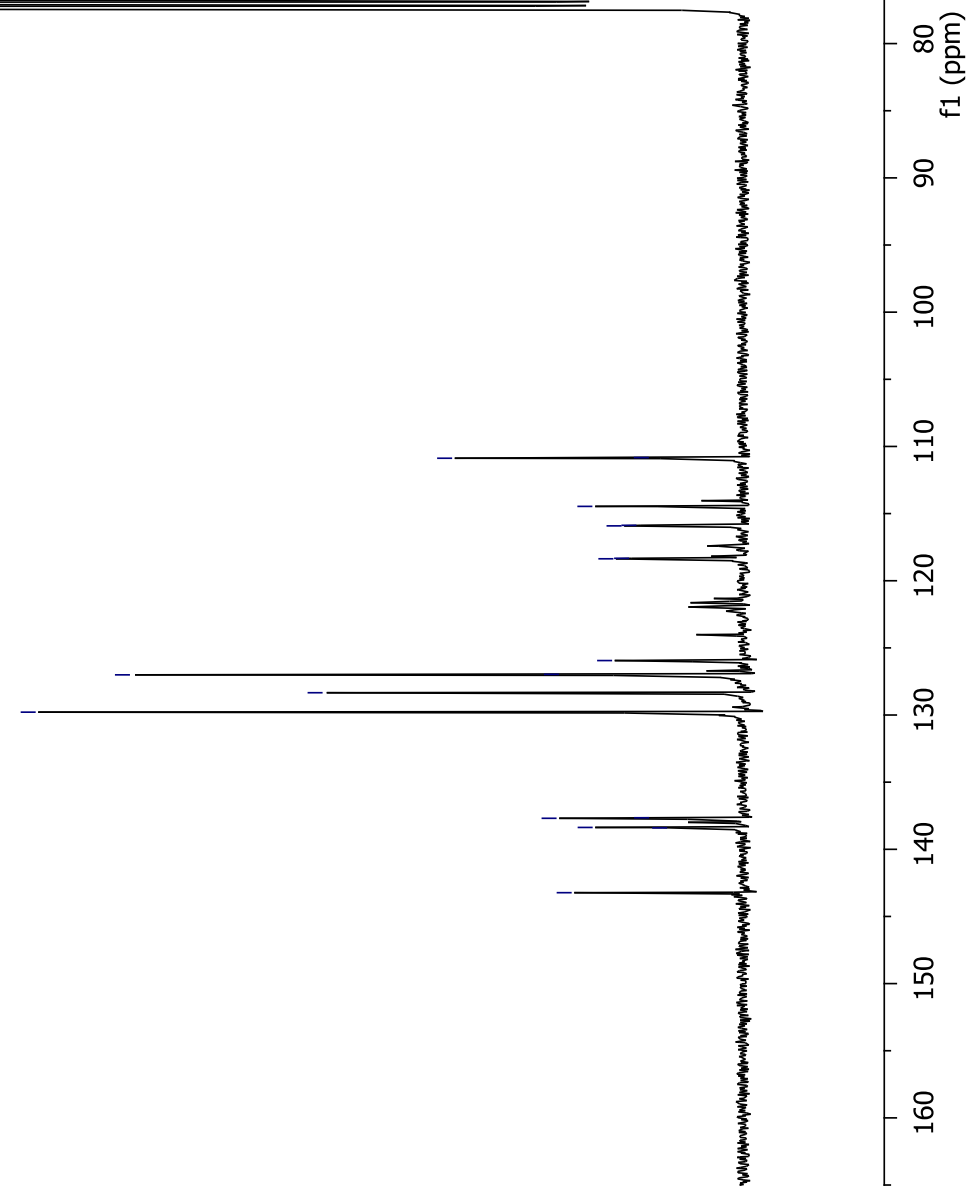



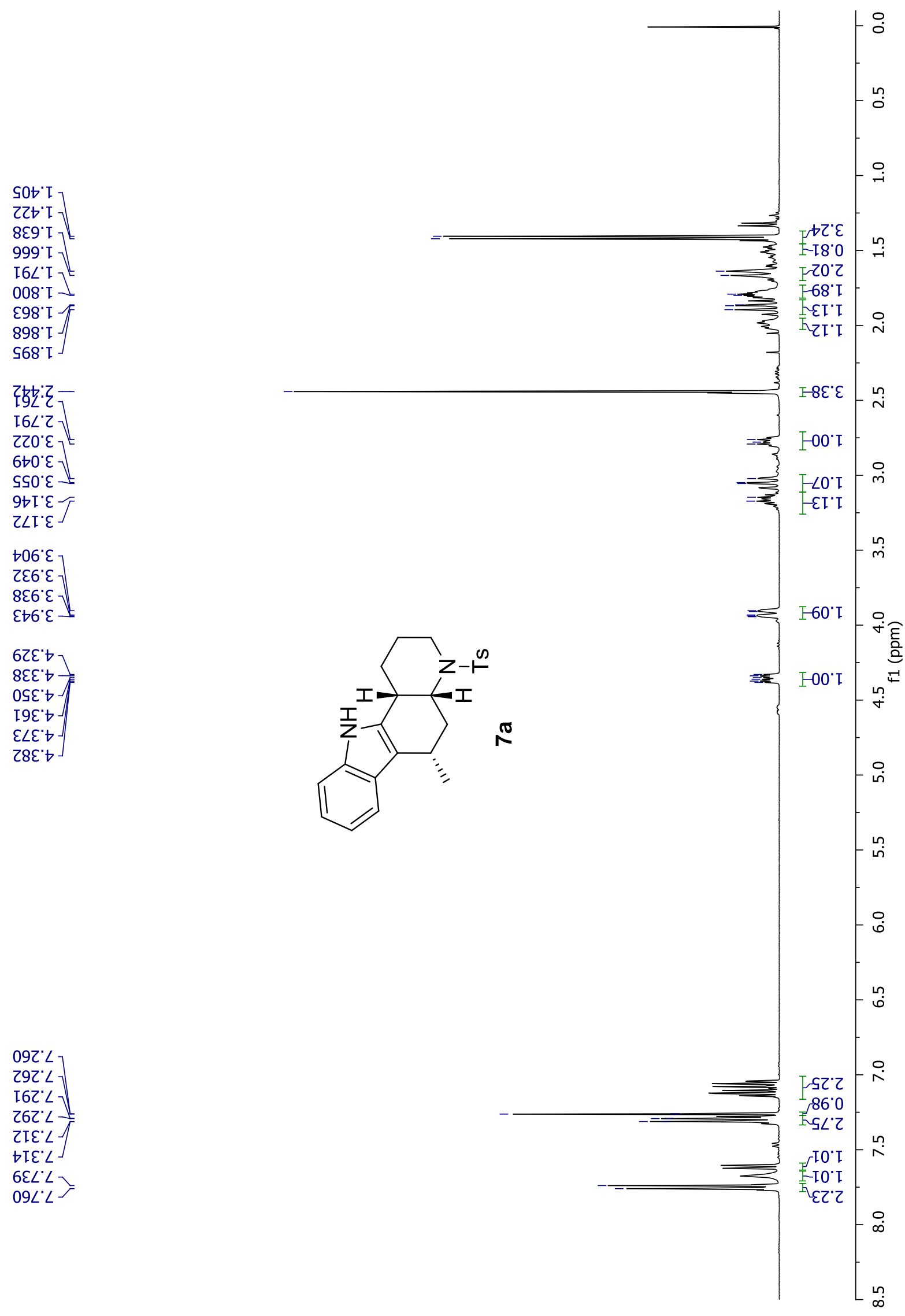
$8 \angle{ }^{\circ}$ 'I 0ZS' IZ

0St $\downarrow 2-$

$0 \rightarrow I^{\circ} 8 Z$

IZE' 82

$8 \angle{ }^{\prime} 乙 \varepsilon-$

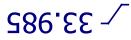

IES.0t -

$6+t \cdot 25-$

$289^{\circ} 9 L$

$000^{\circ} \angle L$

8 [I. $\angle L$

00LOII -

โOI'EI I -

tSZ'6I I

I $\angle 88^{\circ} 6 \mathrm{II}-$

8ЬE'IZI

IS6.9ZI -

$8 \mathrm{I} \angle{ }^{\circ} 6 Z \mathrm{~L}$ -

8IT'9عI

ธعદ'9عI

IZS'8EI

$\angle S O^{\circ} \mathcal{E} \sqcup \mathrm{I}$ -

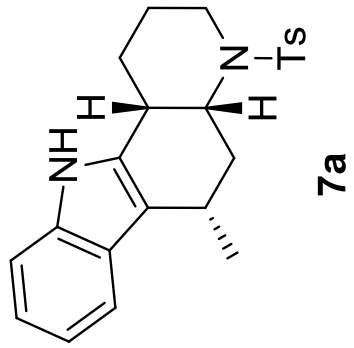

ก

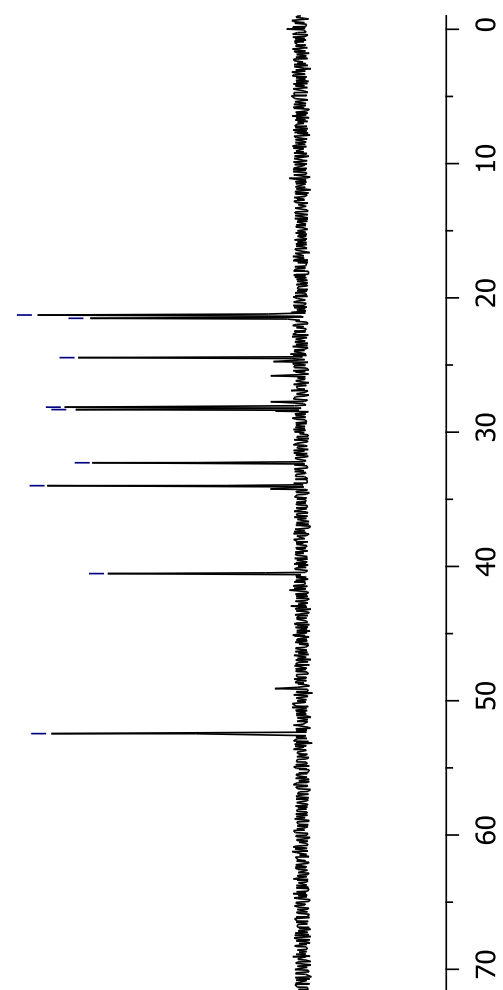



हㅡㅁ

피

8

8

옥

ิㅗㄱ

요

g

옥

$\stackrel{\circ}{\circ}$ 


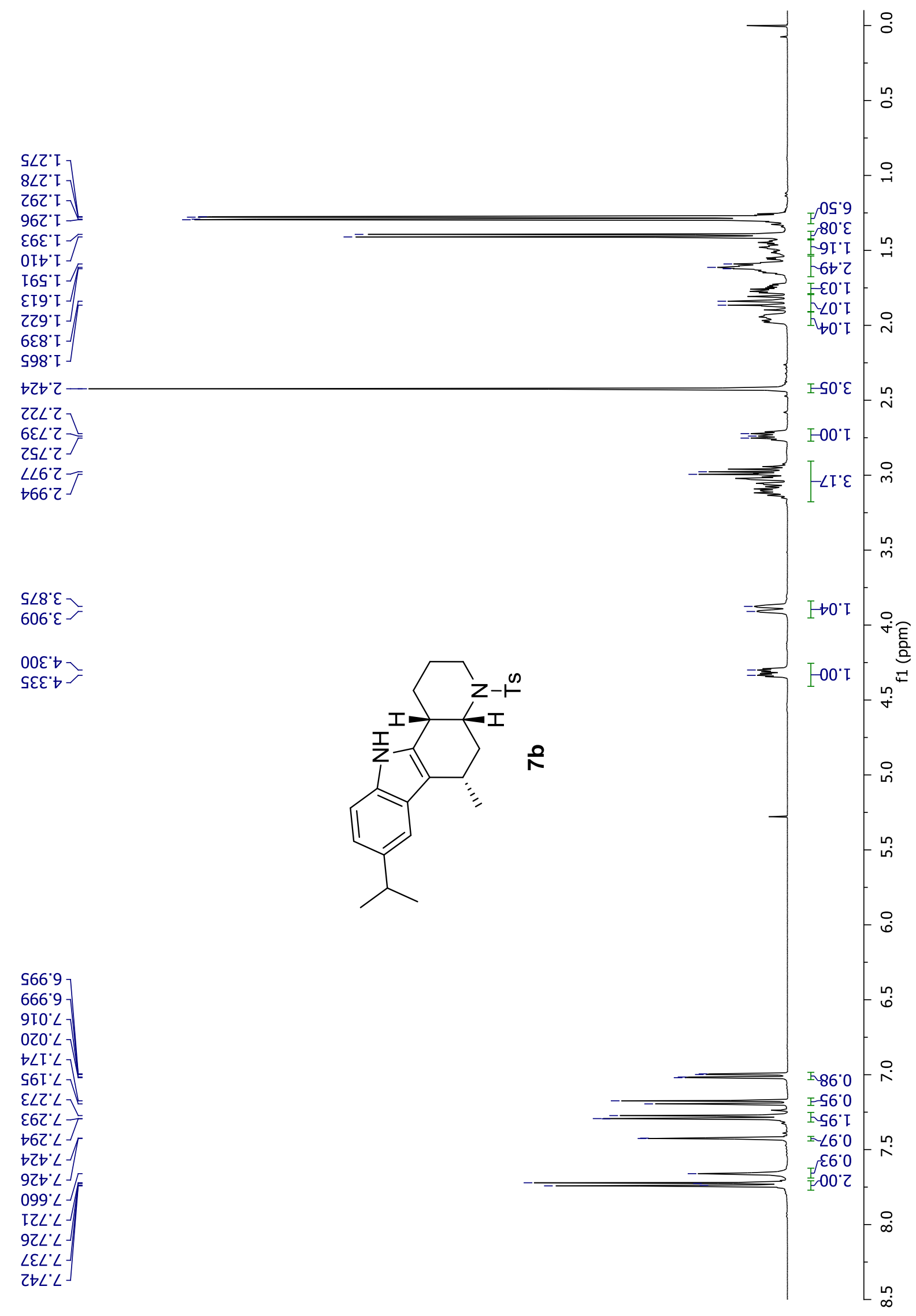



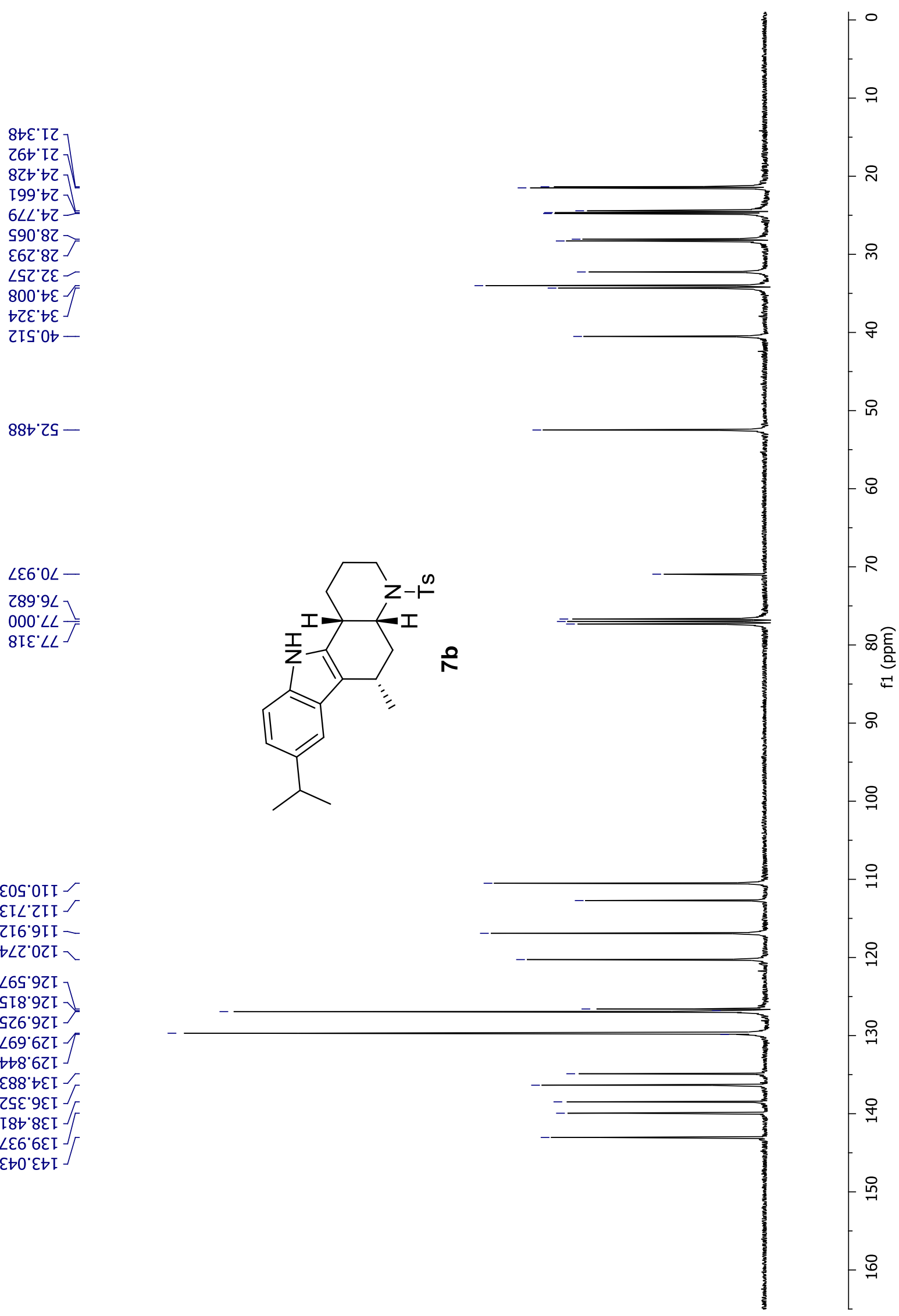

$\angle \varepsilon 6^{\circ} 0 \angle-$ $289^{\circ} 9 L$

$000^{\circ} \angle L$

8 IE $\angle L\rfloor$

EOS'0II -

EI $\angle$ 'ZII $J$

2I6.9IT -

$\checkmark \angle Z^{\circ} 0 Z I$

$\angle 6 S^{\circ} 9 Z \mathrm{I}$

SI8.9ZI

SZ6"9ZI

$\angle 69^{\circ} 6 Z \mathrm{I}-1$

t७8.6ZI

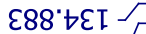

乙S६'9عI

โ8t' $8 \varepsilon[$

$\angle \varepsilon 6^{\circ} 6 \varepsilon \mathrm{I}$

$\varepsilon \mapsto 0^{\circ} \varepsilon \multimap[$

signal at $70.9 \mathrm{ppm}$ being parasitic signal from the NMR machine 
$2000^{\circ}-$

06ع' $\mathrm{I}$

60t:

ยร8. $\mathrm{T}$

6S8. I

t98. $\mathrm{I}-$

โ68. I

9et' $2>$

蛙:

$\angle S L ' Z$

$0 Z 0^{\circ} \varepsilon$

$870^{\circ} \varepsilon$

$\rightarrow S 0^{\circ} \varepsilon$

9ZI' $\varepsilon-$

ZSI' $\varepsilon$

乙† $8^{\circ} \varepsilon$

$\angle 68^{\circ} \varepsilon$

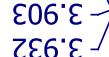

ZTE'॰

โZะ'॰-

$\downarrow \varepsilon \varepsilon^{\prime} \triangleright-$

$t \leftarrow \varepsilon \cdot t$

9SE' $t-$

s9ع. $\downarrow$

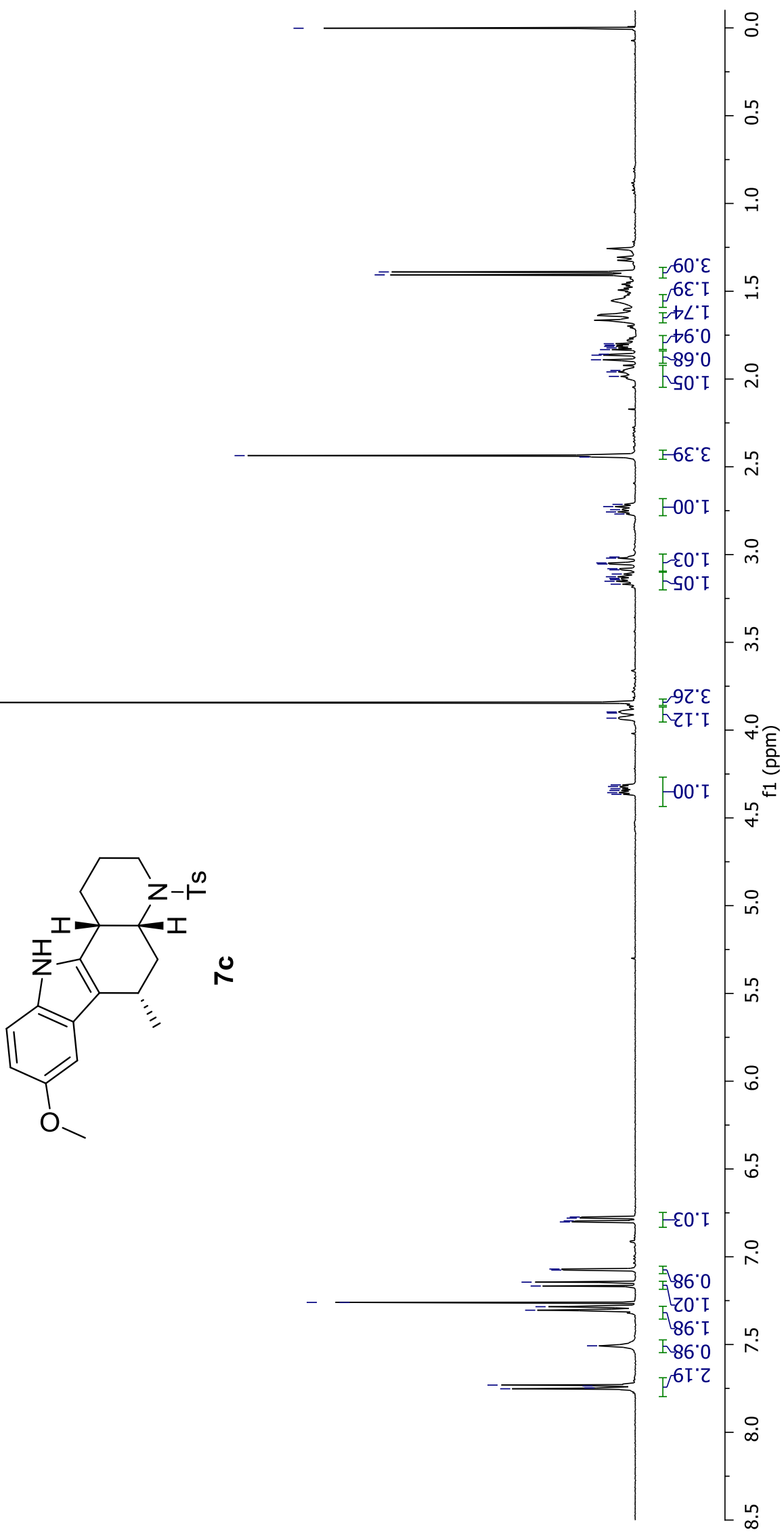

$\forall \angle L ' 9$

$08 \angle ' 9$

96L' 9

$208: 9$

$9 \angle 0^{\circ} \angle 1$

$S \rightarrow I: \angle-$

$\angle 9 I^{\circ} \angle A$

$092^{\circ} \angle$

โ9Z' $\angle$ -

$S 8 Z^{\circ} \angle$

SOE. $\angle]$

โE $L$ ' $\angle$ -

$9 \varepsilon L ' L$

ZS $L L$ 
ESI'IZ

SZS'IZ

9Zち $九 2-$

ISI' $8 Z 7$

乙๖乙 $8 乙$

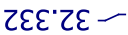

$280^{\circ} \downarrow \varepsilon \digamma$

tIS'0t-

86ع'ZS -

690.95 -

$\varepsilon 89^{\circ} 9 L$

8IE $\angle L$

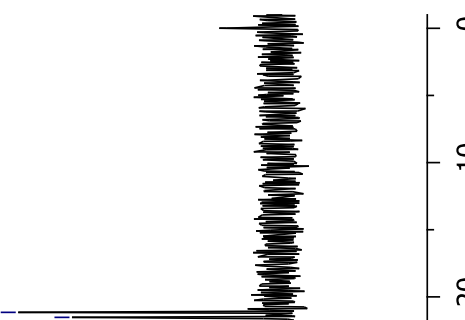

으

ำ

요

q

웅

8

R

हㅡㅁ

$\stackrel{2}{5}$

908'20I -

Zt8.0II

S6I'III

IL6'ZII $\digamma$

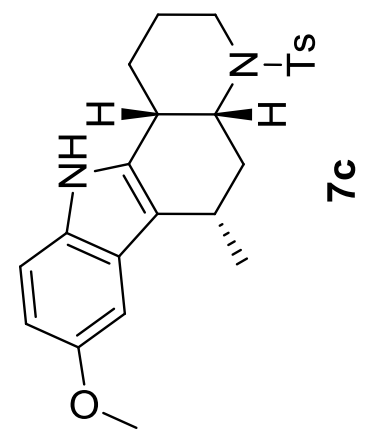

เ96.9ZI

$600^{\circ} \angle Z I-$

II $\angle$ ' 6 ZI

$6^{\circ}$ IEI 5

III' $\angle E I$ -

Z9S'8عโ $厂$

ऽะ0'Е† -

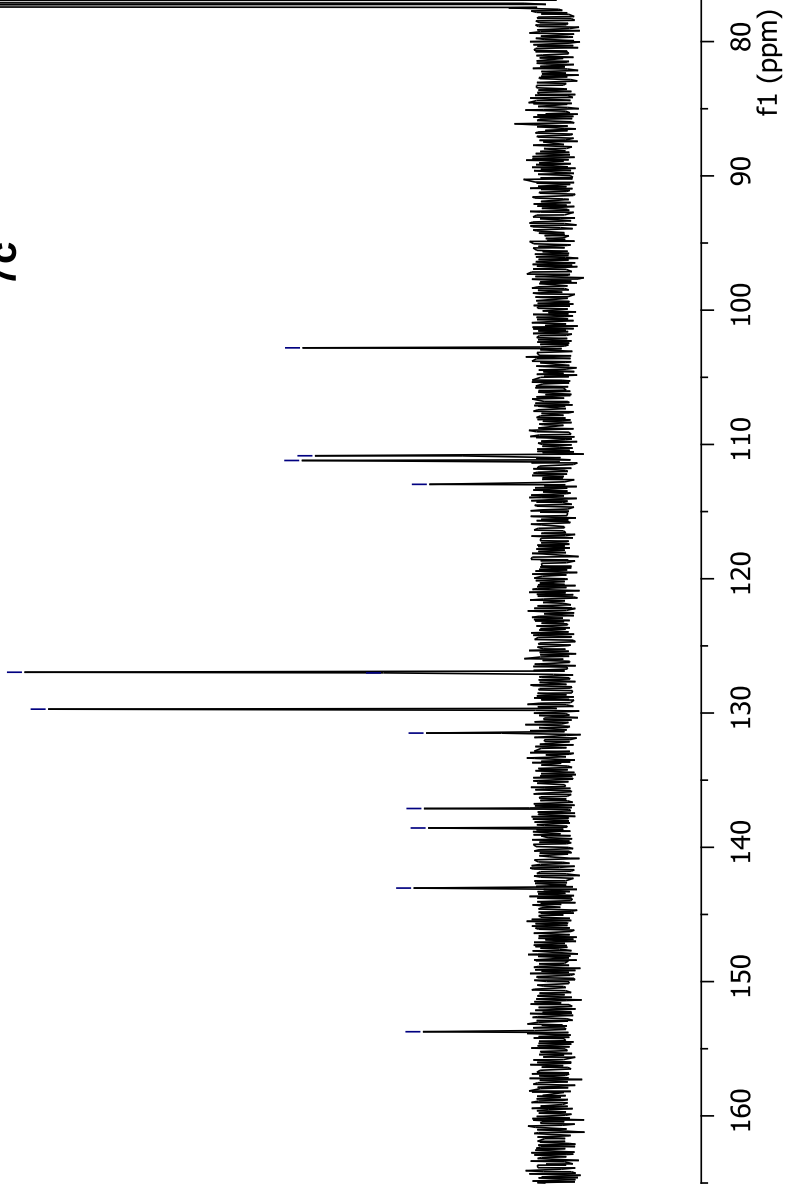

†EL'ESI - 


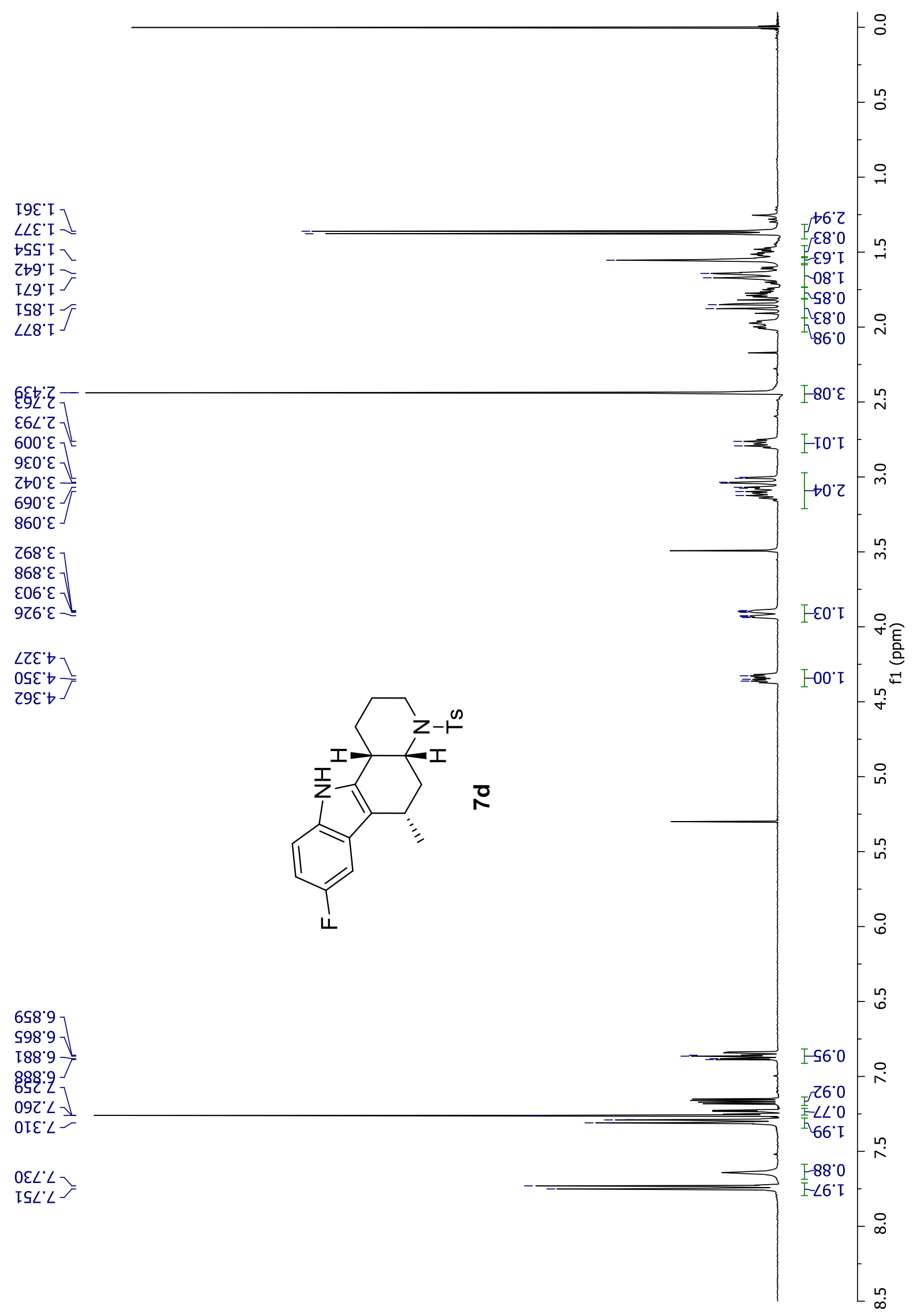


S00. I乙 乙

OES 'IZ -

ZSt" $\downarrow$ -

III'8Z

$\left.\varepsilon \angle I^{\prime} 8 Z\right]$

9เI'Zع-

IOI' $\downarrow \varepsilon-\digamma$

t8t $0 t-$

$\angle 6 Z^{\circ} Z S$ -

$786^{\circ} 0 L$

$289^{\circ} 9 L$

6 IE'LL J

$9\left[6^{\circ} \circ 0[\right.$

$\triangle S I ' S O I-$

โ82.60I

0†S'60I

๑ZO'III-

$\left.\begin{array}{l}\text { ZZІ'LII } \\ 86 \varepsilon^{\circ} \varepsilon I I\end{array}\right]$

乙ह8.9ZI

9S6.9ZI

8EL'6ZI $\leftrightharpoons$

96L'Zعโ $ノ$

ZOI'8ET -

[6t.8Eโ

†OI' $E \triangleright I-$

$\angle t \varepsilon^{\prime} 9 S T$ -

0L9'8S I -
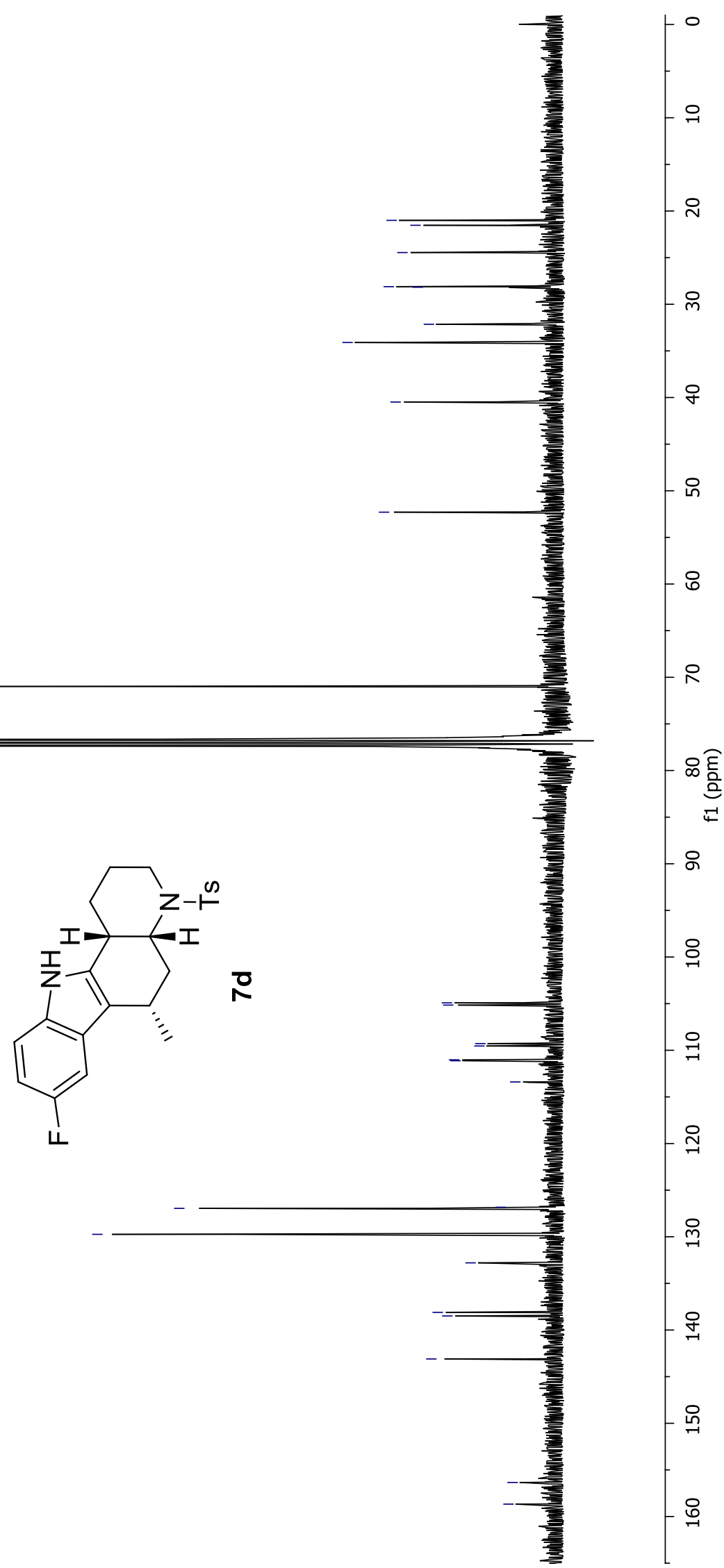

signal at $70.9 \mathrm{ppm}$ being parasitic signal from the NMR machine 


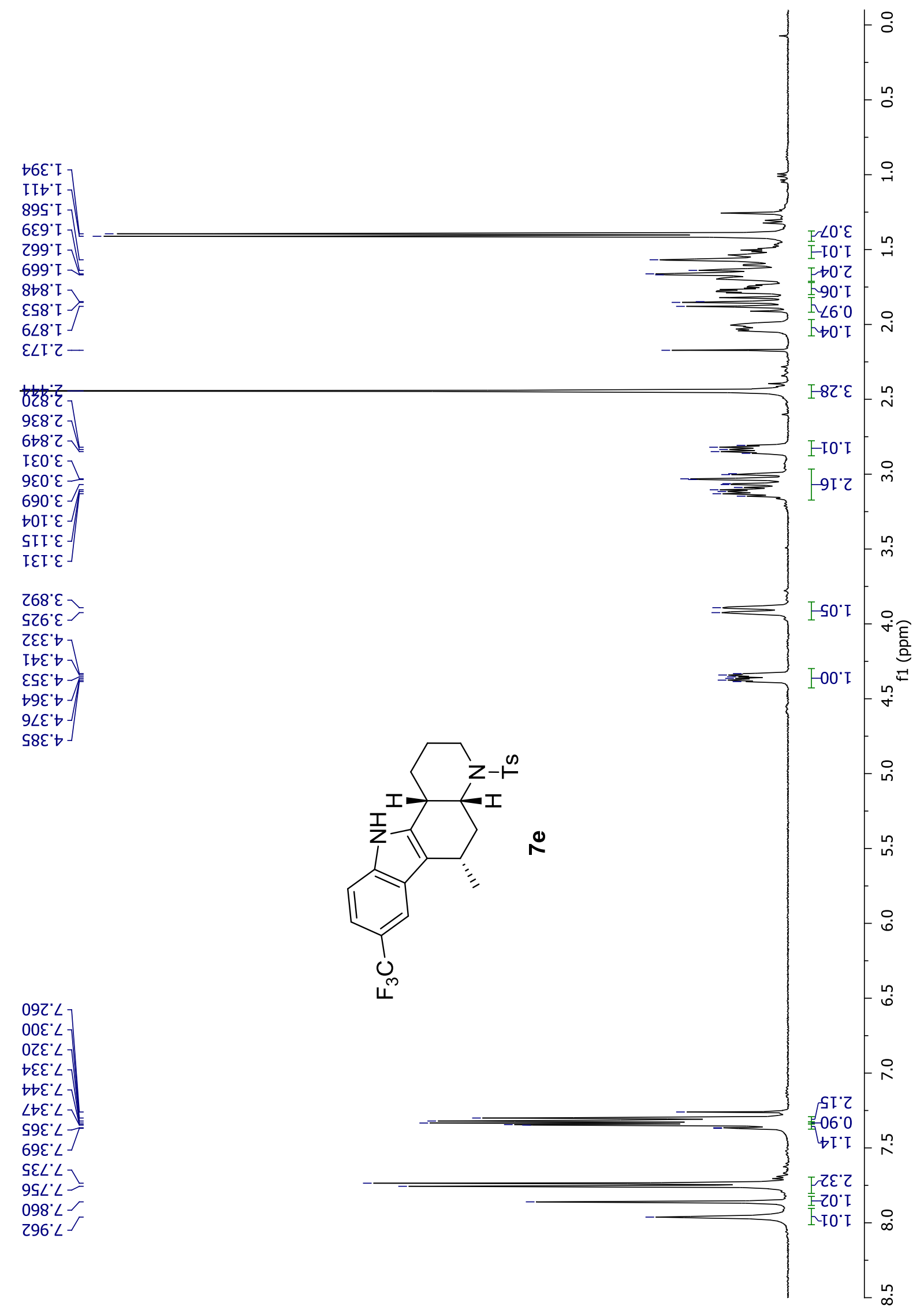


乙2て'น乙

E†S' IZ -

$t \angle t^{\circ} \circ Z-$

$\varepsilon 90.827$

LZI'8Z

$\angle S 6^{\circ}$ 'IE

乙90 $\downarrow \varepsilon \digamma$

69t0 $0 t-$

ธย乙'ZS -
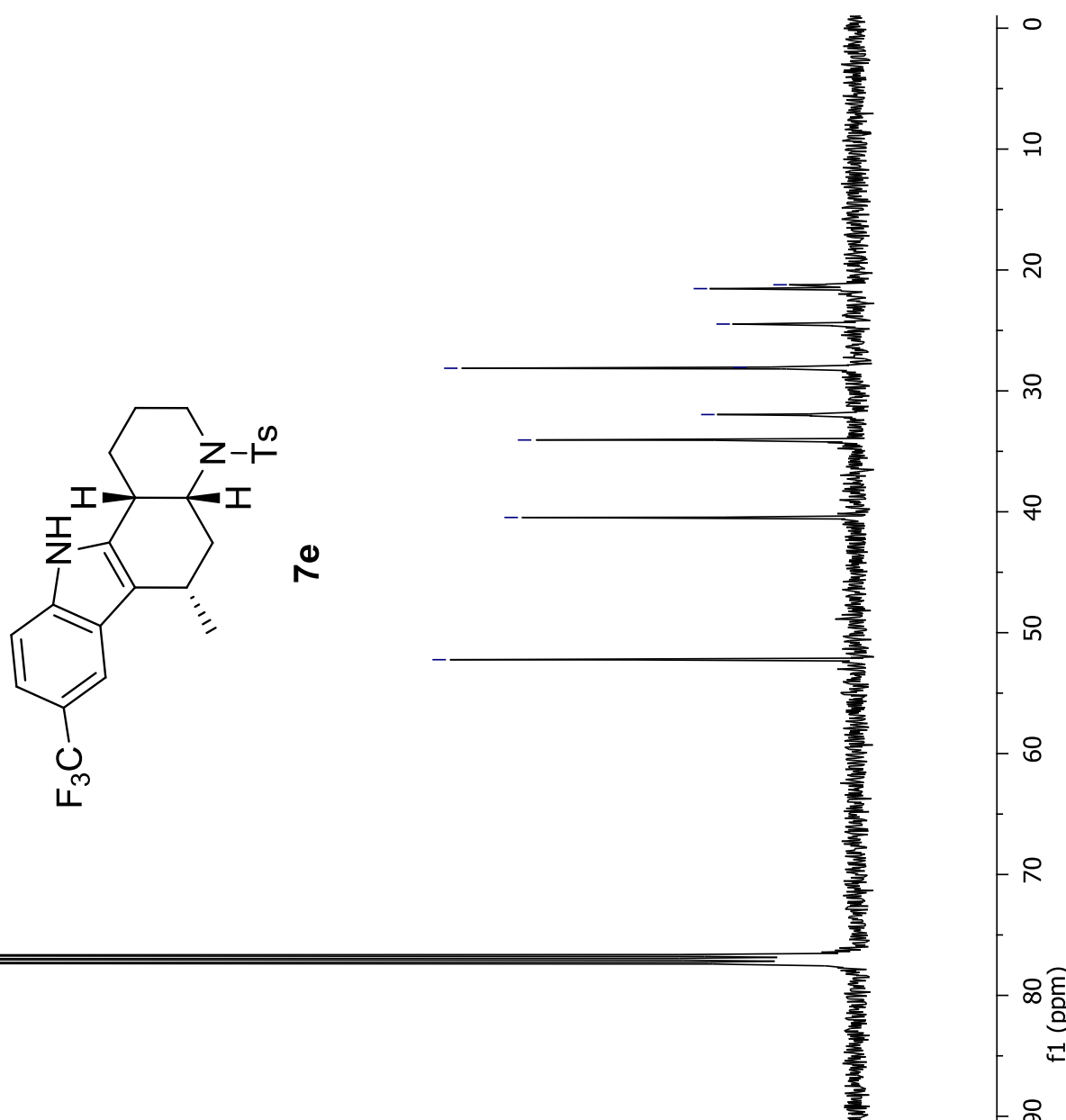

OIT

$\downarrow \angle 6^{\circ} \varepsilon I I$

ОहE' $\angle I I$

ZLE' $\angle I I$

6II'8I I

ZSI'8II

69I'IZI

ZIE' IZI

ह8Ь" IZI

$\angle 6 L^{\prime}$ IZI

ZII'ZZI ]

SI0"ナZI]

600"9ZI

$\varepsilon T \angle ' 9 Z I$

0t6.9ZI

†It'6ZI-

8LL'6ZI

$\angle S L ' \angle E I$

$\varepsilon 66^{\circ} \angle \varepsilon I$

ع9ع $8 \varepsilon \mathrm{I}-$

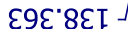

9IZ'E†

Z68'†SI

Z68'†SI

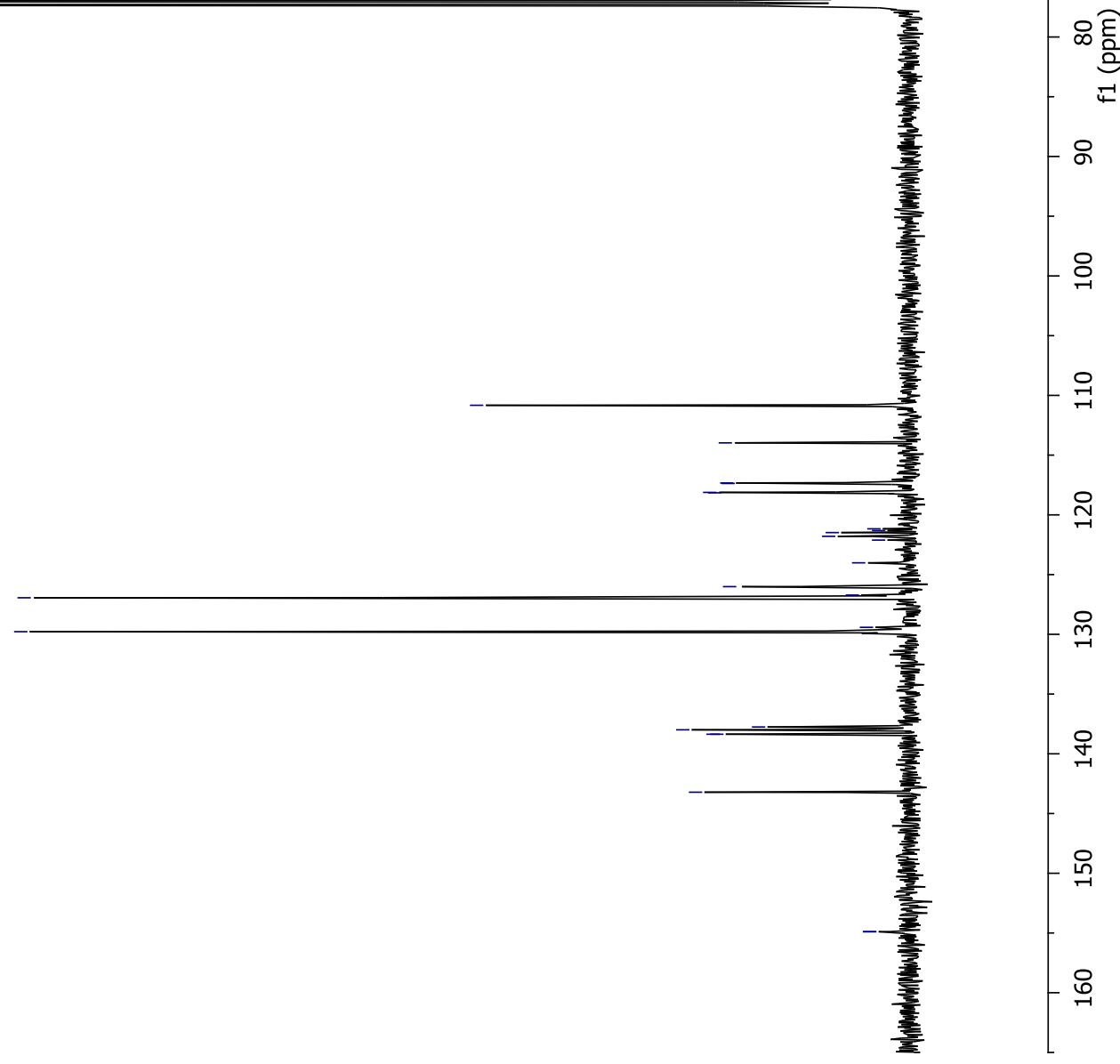




\section{X-Ray Crystallographic Data}

X-Ray crystallographic Data for compound 4a

CCDC 1476675 contain the supplementary crystallographic data for this paper. These data can be obtained free of charge from the Cambridge Crystallographic Data Centre via www.ccdc.cam.ac.uk/data_request/cif

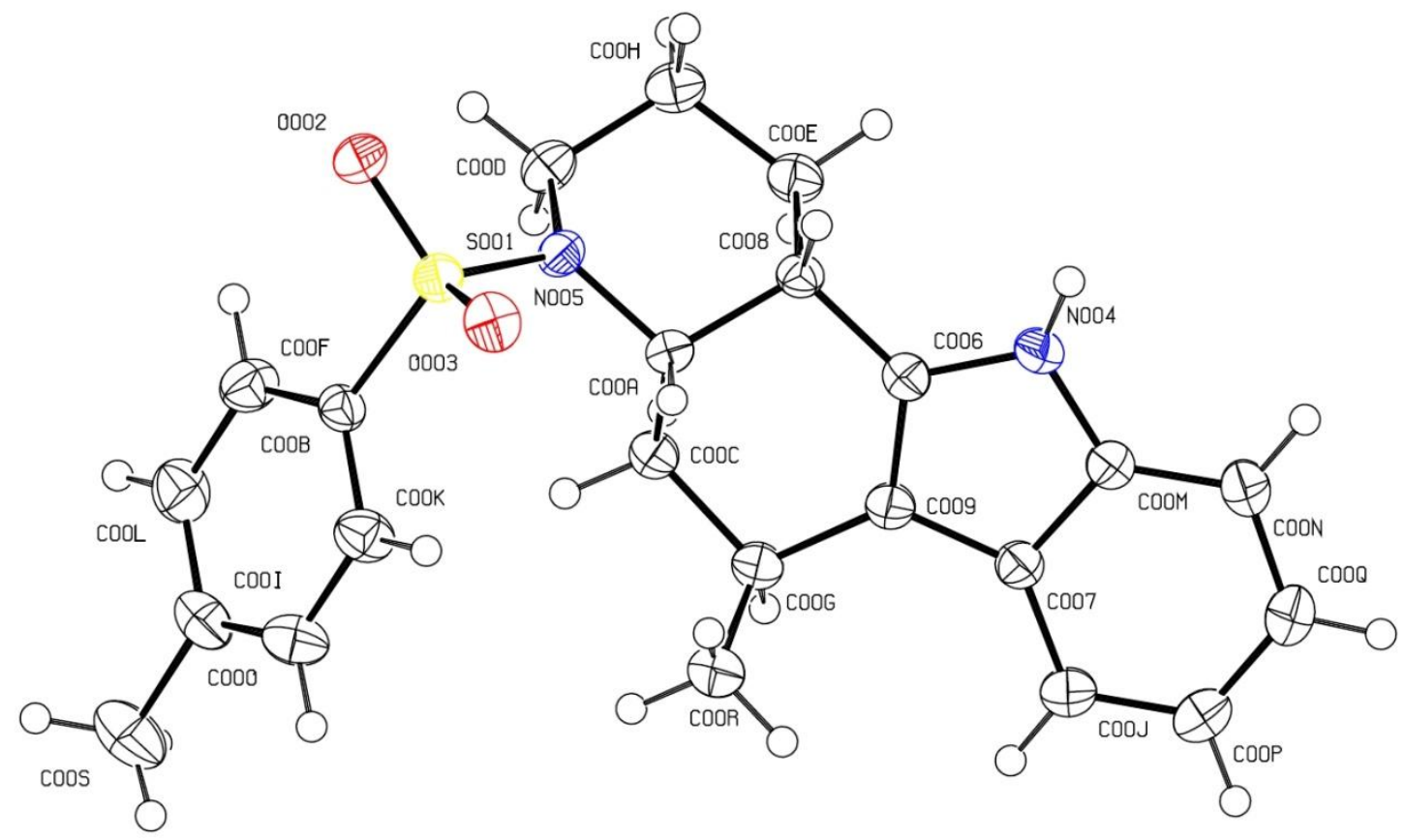

Table 3. Crystal data and structure refinement for $\mathbf{4 a}$.

Identification code

Empirical formula

Formula weight

Temperature

Wavelength

Crystal system

Space group

Unit cell dimensions

Volume

Z

Density (calculated)

Absorption coefficient

$\mathrm{F}(000)$

Crystal size

Theta range for data collection p1585c

$\mathrm{C} 23 \mathrm{H} 26 \mathrm{~N} 2 \mathrm{O} 2 \mathrm{~S}$

394.52

$208 \mathrm{~K}$

$0.71073 \AA$

Monoclinic

P 21/c (No. 14)

$a=14.776(3) \AA$

$\alpha=90^{\circ}$.

$b=9.354(2) \AA$

$\beta=94.17(3)^{\circ}$.

$c=14.360(3) \AA$ $\gamma=90^{\circ}$.

1979.5(7) $\AA^{3}$

4

$1.324 \mathrm{Mg} / \mathrm{m}^{3}$

$0.185 \mathrm{~mm}^{-1}$

840

$0.07 \times 0.25 \times 0.53 \mathrm{~mm}^{3}$

2.6 to $27.5^{\circ}$. 
Table 4. Atomic coordinates $\left(\times 10^{4}\right)$ and equivalent isotropic displacement parameters $\left(\AA^{2} \times 10^{3}\right)$ for p1585c. Parameters of the non-Hydrogen atoms for: p1585c $\quad P$ 21/c $\quad R=0.05$ $\mathrm{U}(\mathrm{eq})$ is defined as one third of the trace of the orthogonalized $\mathrm{U}^{\mathrm{ij}}$ tensor.

\begin{tabular}{|c|c|c|c|c|}
\hline & $x$ & $y$ & z & $\mathrm{U}(\mathrm{eq})$ \\
\hline S001 & $0.40290(3)$ & $0.77954(6)$ & $0.57944(3)$ & $0.0259(1)$ \\
\hline 0002 & $0.48372(8)$ & $0.86539(16)$ & $0.59223(9)$ & $0.0326(4)$ \\
\hline 0003 & $0.38857(9)$ & $0.66776(15)$ & $0.64496(8)$ & $0.0318(4)$ \\
\hline N004 & $0.33093(11)$ & $0.24063(19)$ & $0.33126(11)$ & $0.0282(5)$ \\
\hline N005 & $0.40455(10)$ & $0.70656(17)$ & $0.47755(10)$ & $0.0250(5)$ \\
\hline C006 & $0.31249(12)$ & $0.3774(2)$ & $0.36039(12)$ & $0.0245(6)$ \\
\hline C007 & $0.17961(12)$ & $0.2719(2)$ & $0.31596(12)$ & $0.0265(6)$ \\
\hline COOB & $0.38393(11)$ & $0.4809(2)$ & $0.39469(12)$ & $0.0225(6)$ \\
\hline CO09 & $0.22112(12)$ & $0.4012(2)$ & $0.35133(12)$ & $0.0261(6)$ \\
\hline COOA & $0.33633(11)$ & $0.5964(2)$ & $0.44869(12)$ & $0.0240(6)$ \\
\hline $\mathrm{COOB}$ & $0.30839(12)$ & $0.8933(2)$ & $0.57866(12)$ & $0.0245(6)$ \\
\hline $\mathrm{COOC}$ & $0.25294(12)$ & $0.6550(2)$ & $0.39099(13)$ & $0.0283(6)$ \\
\hline COOD & $0.44791(13)$ & $0.7774(2)$ & $0.40104(13)$ & $0.0297(6)$ \\
\hline COOE & $0.43421(12)$ & $0.5454(2)$ & $0.31441(12)$ & $0.0289(6)$ \\
\hline $\mathrm{COOF}$ & $0.31183(14)$ & $1.0302(2)$ & $0.54148(13)$ & $0.0339(7)$ \\
\hline COOG & $0.17858(12)$ & $0.5410(2)$ & $0.37577(14)$ & $0.0309(6)$ \\
\hline $\mathrm{COOH}$ & $0.49788(12)$ & $0.6652(2)$ & $0.34870(13)$ & $0.0308(6)$ \\
\hline $\mathrm{COOI}$ & $0.15359(14)$ & $1.0631(3)$ & $0.56712(13)$ & $0.0348(7)$ \\
\hline COOJ & $0.09007(13)$ & $0.2272(2)$ & $0.29436(13)$ & $0.0339(7)$ \\
\hline COOK & $0.22838(13)$ & $0.8419(2)$ & $0.61037(13)$ & $0.0332(7)$ \\
\hline $\mathrm{COOL}$ & $0.23504(15)$ & $1.1133(2)$ & $0.53682(14)$ & $0.0388(7)$ \\
\hline COOM & $0.25027(13)$ & $0.1735(2)$ & $0.30409(13)$ & $0.0279(6)$ \\
\hline COON & $0.23431(14)$ & $0.0357(2)$ & $0.27137(14)$ & $0.0372(7)$ \\
\hline $\mathrm{COOO}$ & $0.15184(14)$ & $0.9269(3)$ & $0.60395(13)$ & $0.0370(7)$ \\
\hline COOP & $0.07417(14)$ & $0.0894(3)$ & $0.26361(15)$ & $0.0405(7)$ \\
\hline $\mathrm{COOQ}$ & $0.14492(14)$ & $-0.0050(3)$ & $0.25177(14)$ & $0.0419(8)$ \\
\hline COOR & $0.12054(14)$ & $0.5294(3)$ & $0.45857(16)$ & $0.0467(8)$ \\
\hline COOS & $0.06945(15)$ & $1.1552(3)$ & $0.55820(16)$ & $0.0512(9)$ \\
\hline
\end{tabular}

